\title{
Igneous garnet and amphibole fractionation in the roots of island arcs: experimental constraints on andesitic liquids
}

\author{
Raquel Alonso-Perez • Othmar Müntener • \\ Peter Ulmer
}

Received: 23 January 2008/Accepted: 6 October 2008/Published online: 30 October 2008

(C) Springer-Verlag 2008

\begin{abstract}
To evaluate the role of garnet and amphibole fractionation at conditions relevant for the crystallization of magmas in the roots of island arcs, a series of experiments were performed on a synthetic andesite at conditions ranging from 0.8 to $1.2 \mathrm{GPa}, 800-1,000^{\circ} \mathrm{C}$ and variable $\mathrm{H}_{2} \mathrm{O}$ contents. At water undersaturated conditions and $f \mathrm{O}_{2}$ established around QFM, garnet has a wide stability field. At 1.2 GPa garnet + amphibole are the high-temperature liquidus phases followed by plagioclase at lower temperature. Clinopyroxene reaches its maximal stability at $\mathrm{H}_{2} \mathrm{O}$ contents $\leq 9 \mathrm{wt} \%$ at $950^{\circ} \mathrm{C}$ and is replaced by amphibole at lower temperature. The slopes of the plagioclase-in boundaries are moderately negative in $\mathrm{T}-\mathrm{X}_{\mathrm{H}_{2} \mathrm{O}}$ space. At $0.8 \mathrm{GPa}$, garnet is stable at magmatic $\mathrm{H}_{2} \mathrm{O}$ contents exceeding $8 \mathrm{wt} \%$ and is replaced by spinel at decreasing dissolved $\mathrm{H}_{2} \mathrm{O}$. The liquids formed by crystallization evolve through continuous silica increase from andesite to dacite and rhyolite for the 1.2 GPa series, but show substantial enrichment in $\mathrm{FeO} / \mathrm{MgO}$ for the $0.8 \mathrm{GPa}$ series related to the contrasting roles of garnet and amphibole in fractionating $\mathrm{Fe}-\mathrm{Mg}$ in derivative liquids. Our experiments indicate that the stability of igneous garnet increases with increasing dissolved $\mathrm{H}_{2} \mathrm{O}$ in silicate liquids and is thus likely to affect trace element compositions of $\mathrm{H}_{2} \mathrm{O}$-rich derivative arc volcanic rocks by fractionation. Garnetcontrolled trace element ratios cannot be used as a proxy
\end{abstract}

Communicated by T. L. Grove.

R. Alonso-Perez · P. Ulmer

Institute of Mineralogy and Petrology, ETH, Zurich, Switzerland

O. Müntener $(\bowtie)$

Institute of Mineralogy and Geochemistry,

University of Lausanne, Lausanne, Switzerland

e-mail: othmar.muntener@unil.ch for 'slab melting', or dehydration melting in the deep arc. Garnet fractionation, either in the deep crust via formation of garnet gabbros, or in the upper mantle via formation of garnet pyroxenites remains an important alternative, despite the rare occurrence of magmatic garnet in volcanic rocks.

Keywords Experimental petrology · Hydrous andesite liquids · High-pressure crystallization - Amphibole . Garnet fractionation

\section{Introduction}

Major and trace element similarities between estimates of the composition of the continental crust and calc-alkaline andesites indicate that subduction zones are one of the key areas in the generation of new continental crust (Gill 1981; Kay and Kay 1985; Kelemen et al. 2003; Rudnick 1995). A major question of continental crust formation is if and how fractionation of primary arc magmas could ultimately produce Si-rich derivative liquids that resemble the andesitic composition of continental crust (Taylor 1967). Major and trace element concentrations of volcanic and plutonic calc-alkaline differentiates in continental margin settings (e.g. Alps, Andes, Himalayas and Alaska) indicate that silica-poor minerals such as garnet, amphibole and $\mathrm{Fe}-$ Ti-oxides played a significant role during differentiation of basaltic liquids (Jan and Howie 1981; Ulmer et al. 1985; DeBari and Coleman 1989; Greene et al. 2006). Crystallization and fractionation of garnet at high pressure to generate high-silica calc-alkaline liquids has originally been proposed by Trevor Green and coworkers (Green 1972; Green and Ringwood 1968a, b), based on the occurrence of garnet-phenocrysts in calc-alkaline dacites 
and on experimental phase equilibria on a series of calcalkaline compositions (high-Al olivine tholeiite, high-Al qtz-tholeiite, basaltic andesite, andesite, dacite and rhyodacite). However, there is limited experimental data that have systematically investigated the influence of variable amounts of $\mathrm{H}_{2} \mathrm{O}$ (and other volatiles) on the relative stabilities of garnet, amphibole, pyroxene and plagioclase in basaltic to andesitic liquids at conditions relevant for the lower arc crust (Green 1992; Müntener et al. 2001). Magmatic garnet has been described from andesitic to dacitic calc-alkaline volcanics (Day et al. 1992; Evans and Vance 1987; Fitton 1972; Harangi et al. 2001) indicating that high-pressure garnet crystallization and fractionation at a depth of $25-45 \mathrm{~km}$ is an important process in the generation of these lavas.

The potential localities and processes, where garnet might be involved in the petrogenesis of subduction-related magmas are schematically illustrated in Fig. 1. Four potential areas can be identified: (a) garnet fractionation at high pressure at the base of arc crust (Müntener and Ulmer 2006), (b) partial melting of mafic rocks at the base of arc crust, leaving a garnet-bearing granulitic residue (Atherton and Petford 1993), (c) partial melting of a garnet-lherzolite source (Ulmer 1989a) and melting of the subducted slab, leaving a rutile-bearing eclogite residue (Defant and Drummond 1990). Moreover, the occurrence of garnetbearing ultramafic to mafic plutonic rocks in deep arc crustal sections and at the crust-mantle boundary (Kohistan arc, Pakistan: Burg et al. 1998; Jan and Howie 1981; Ringuette et al. 1999), south-central Alaskan Tonsina Complex (DeBari and Coleman 1989; Greene et al. 2006), as well as studies from crust and mantle xenoliths (Ducea and Saleeby 1996, 1998) provides compelling evidence that garnet fractionation/crystallization from basaltic magmas is a potentially important process in the differentiation of subduction-related magmas. The field observations that many of these terrains show cumulate

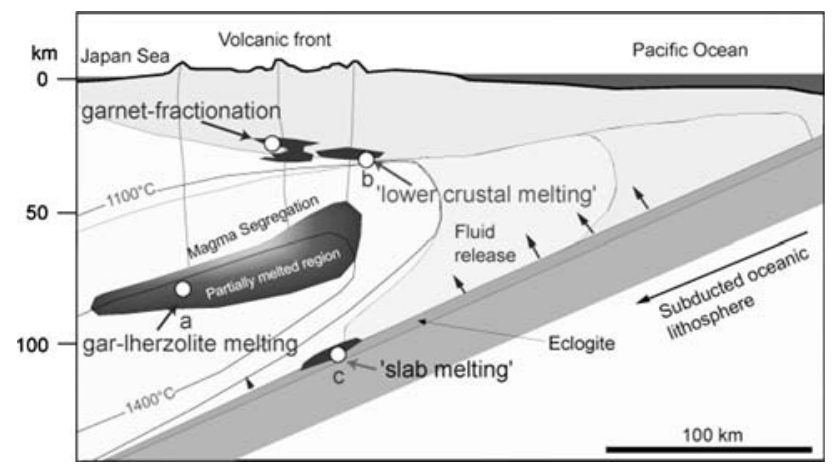

Fig. 1 Potential localities/processes where garnet could be involved in the genesis of andesitic magmas at convergent-plate margins (basic diagram modified after Kushiro 1987). Thin black lines are isotherms. See text for discussion sequences support the important role of fractionation at high-pressure. The importance of amphibole fractionation in the genesis of calc-alkaline rocks was already proposed by Bowen (1928) and has been a hotly debated topic ever since (Allen et al. 1975; Cawthorn and O'Hara 1976; Green and Ringwood 1968b; Huang and Wyllie 1986; Sisson and Grove 1993a; Davidson et al. 2007). This debate has been revived in recent times, since detailed trace element partitioning studies advocate an important role of amphibole in fractionating geochemical 'twins', such as $\mathrm{Nb}$ and $\mathrm{Ta}$ (Blundy and Wood 2003; Tiepolo et al. 2000). The role of magnetite in the formation of calc-alkaline magmas was first discussed by Osborn (1959). He proposed that the lack of iron-enrichment in the calc-alkaline trend is caused by early fractionation of magnetite and is thus primarily controlled by oxygen fugacity. Clearly, differentiation of $\mathrm{H}_{2} \mathrm{O}$ undersaturated magmas, under conditions relevant for the roots of island arc crust is a key for the understanding of the genesis of andesites and dacites, and thus for the continental crust in general.

Here, we present the results of a phase equilibria study on a $\mathrm{H}_{2} \mathrm{O}$-undersaturated andesitic composition in the temperature range of $800-1,000^{\circ} \mathrm{C}$, pressures of $0.8-1.2 \mathrm{GPa}$, $\mathrm{H}_{2} \mathrm{O}$ contents of 4,6 and $8 \mathrm{wt} \%$ and $f \mathrm{O}_{2}$ between $\sim \mathrm{QFM}$ and $\mathrm{Ni}-\mathrm{NiO}$. We discuss the relative roles of garnet, amphibole, pyroxene and plagioclase on derivative, $\mathrm{H}_{2} \mathrm{O}$-undersaturated liquids in order to explore the effects of variable $\mathrm{H}_{2} \mathrm{O}$ content on andesite phase equilibria under conditions relevant for arc crust formation. Finally, we will critically evaluate previous proposals invoking partial melting of the lower crust, or melting of the subducting slab (e.g. 'slab melting') as the dominant mechanisms in explaining geochemical peculiarities of subduction zone magmas.

\section{Experimental design and analytical techniques}

A synthetic andesite composition was used as starting material for this study (Table 1). The andesite composition was derived by a series of fractional crystallization experiments (Kägi 2000) conducted on a natural picrobasalt from the Adamello batholith, N-Italy (Ulmer 1986, 1989b). We remixed the derivative andesite from natural silicates, synthetic oxides and hydroxides in order to achieve variable $\mathrm{H}_{2} \mathrm{O}$ contents, of about 4,6 and $8 \mathrm{wt} \%$. To constrain the redox conditions of the experiments close to the $\mathrm{Ni-NiO}$ buffer equilibrium, the "intrinsic" $f \mathrm{O}_{2}$ approach was used (Kägi et al. 2005). The intrinsic $f \mathrm{O}_{2}$ of the starting material was adjusted via the molar $\mathrm{Fe}_{2} \mathrm{O}_{3} / \mathrm{FeO}$ ratio of the starting powder by balancing $\mathrm{Fe}_{2} \mathrm{O}_{3}$ (hematite) and fayalite according to the equation of Kress and Carmichael (1991). We have chosen an initial value of 
Table 1 Starting compositions used in this study

\begin{tabular}{|c|c|c|c|c|c|c|c|c|c|c|c|c|c|}
\hline Starting material & $\mathrm{SiO}_{2}$ & $\mathrm{TiO}_{2}$ & $\mathrm{Al}_{2} \mathrm{O} 3$ & $\mathrm{Fe}_{2} \mathrm{O}_{3}$ & $\mathrm{FeO}_{\text {tot }}$ & $\mathrm{MnO}$ & $\mathrm{MgO}$ & $\mathrm{CaO}$ & $\mathrm{Na}_{2} \mathrm{O}$ & $\mathrm{K}_{2} \mathrm{O}$ & $\mathrm{H}_{2} \mathrm{O}$ & Total & Mg\# \\
\hline F8a-4 & 54.82 & 0.91 & 18.05 & 1.66 & 5.94 & 0.29 & 2.83 & 7.12 & 2.83 & 1.42 & 4.14 & 100 & 0.404 \\
\hline F8a-6 & 53.67 & 0.89 & 17.68 & 1.62 & 5.81 & 0.29 & 2.77 & 6.97 & 2.77 & 1.39 & 6.15 & 100 & 0.404 \\
\hline F8a-8 & 52.53 & 0.87 & 17.30 & 1.59 & 5.69 & 0.28 & 2.71 & 6.82 & 2.71 & 1.36 & 8.14 & 100 & 0.404 \\
\hline
\end{tabular}

Starting compositions correspond to a liquid obtained by Kägi (2000) in fractional crystallization experiments starting from a hydrous picrobasalt at $1.0 \mathrm{GPa}, 1020^{\circ} \mathrm{C}$. Compositions were synthesized with 4,6 and $8 \mathrm{wt} \% \mathrm{H}_{2} \mathrm{O}$, respectively. $\mathrm{Fe}_{2} \mathrm{O}_{3}$ and $\mathrm{FeO}$ adjusted to a molar $\mathrm{Fe}_{2} \mathrm{O}_{3} / \mathrm{FeO}$ ratio of 0.125 corresponding to $\mathrm{Ni}-\mathrm{NiO}+0.6$ at $1,000^{\circ} \mathrm{C}$ and $1.0 \mathrm{GPa}$ according to Kress and Carmichael (1991). $\mathrm{Mg} \# \mathrm{molar}[\mathrm{MgO} /(\mathrm{MgO}+\mathrm{FeO})]$; all $\mathrm{Fe}$ as $\mathrm{Fe}^{2+}$. During a later phase of the study, new starting material was prepared, with identical major element composition, but with $2,000 \mu \mathrm{g} / \mathrm{g}$ trace elements added. These starting compositions are termed $4 \mathrm{c}, 6 \mathrm{c}$ and $8 \mathrm{c}$ in Table 2 , respectively

0.125 , corresponding to a $\log \mathrm{fO}_{2}$ of $-9.35(\mathrm{QFM}+1$ or $\mathrm{NNO}+0.6)$ at $1.0 \mathrm{GPa}$ and $1,000^{\circ} \mathrm{C}$. Firstly, $\mathrm{SiO}_{2}, \mathrm{TiO}_{2}$, $\mathrm{MgO}, \mathrm{CaCO}_{3}$ and $\mathrm{Fe}_{2} \mathrm{O}_{3}$ where weighed out and ground for $1 \mathrm{~h}$ (in order to obtain a grain size of $\leq 10 \mu \mathrm{m}$ ) under ethanol, using an agate mortar and pestle. The resulting powder was fired at $1,000^{\circ} \mathrm{C}$ for $2 \mathrm{~h}$ to decarbonate $\mathrm{CaCO}_{3}$ and drive off adsorbed $\mathrm{H}_{2} \mathrm{O}$. This mixture was weighed after firing to determine whether the decarbonation of the $\mathrm{CaCO}_{3}$ was complete. To this mixture, weighed aliquots of $\mathrm{Fe}_{2} \mathrm{SiO}_{4}, \mathrm{Na}_{2} \mathrm{SiO}_{3}, \mathrm{KAlSi}_{3} \mathrm{O}_{8}, \mathrm{AlOOH}, \mathrm{Al}(\mathrm{OH})_{3}$ and $\mathrm{MnO}$ were added in appropriate proportions, ground for $1 \mathrm{~h}$ under ethanol, using an agate mortar and pestle, and dried and stored at $110^{\circ} \mathrm{C}$. Fayalite and K-feldspar were synthesized following the methods described by Kägi et al. (2005). No liquid $\mathrm{H}_{2} \mathrm{O}$ was added to the starting material.

The experiments were carried out in end-loaded pistoncylinder apparatus using $14 \mathrm{~mm}$ diameter NaCl-Pyrex cells. The capsules were surrounded by alumina and Pyrex glass. A friction correction of $-3 \%$ was applied. The temperature was measured using a type $\mathrm{B} \mathrm{Pt}_{94} \mathrm{Rh}_{6}$, $\mathrm{Pt}_{70} \mathrm{Rh}_{30}$ thermocouple, with an estimated accuracy of $\pm 5^{\circ}$; no corrections for the pressure effects on emf were applied. Switching off the power supply terminated the experiments and resulted in quench rates in the order of $100^{\circ} \mathrm{C} / \mathrm{s}$, which were high enough to quench the liquids to glass. Two different sets of experiments have been carried out: (1) Fepresaturated 'sample-in-sample' double capsule technique and (2) double capsule technique. The double capsule technique was employed to minimize Fe-loss and $\mathrm{H}_{2}$ diffusion and to constrain relatively high $\mathrm{fO}_{2}(\mathrm{~N}-\mathrm{NNO}+1)$. In order to further minimize Fe-loss we pre-saturated the inner capsule $\left(\mathrm{Au}_{90} \mathrm{Pd}_{10} 2.3 \mathrm{~mm}\right.$ outer diameter $)$, which were filled with the identical starting material and held at $1 \mathrm{bar}$ and $1,180^{\circ} \mathrm{C}$ in a vertical gas-mixing furnace for $48 \mathrm{~h}$ at an $\mathrm{fO}_{2}$ of $1 \mathrm{log}$ unit below the FMQ buffer (Gaetani and Grove 1998; Hall et al. 2004; Kägi et al. 2005). Tylan digital gas-flow controllers were used to control the $\mathrm{CO}_{2}-$ $\mathrm{H}_{2}$ gas mixtures. After quenching, the capsules were cleaned in a warm HF bath for $48 \mathrm{~h}$. After leaching, the cleaned Fe-presaturated capsule were filled with the appropriate starting materials, welded shut, and placed into the outer Pt capsule (4.0 mm outer diameter), which contained the same starting material. In some of the presaturated runs, especially those at high temperature, relative Fe-gain was observed (Table 2). Therefore, in subsequent experiments, we decided to use the double capsule technique without the pre-saturation procedure. Relative Fe losses or gains were always lower than $10 \%$ relative (estimates based on mass balance calculations) in all, but one case. Therefore, we assume that a relatively constant bulk composition was maintained during the experiments. $\mathrm{H}_{2} \mathrm{O}$ loss during welding was avoided by freezing the capsule with liquid nitrogen and/or surrounding it with a wet tissue. Weight tests before and after welding indicated no loss of $\mathrm{H}_{2} \mathrm{O}$. Consequently, we are confident that a reasonable approach to closed-system conditions could be achieved in our experiments. Furthermore, we determined the $\mathrm{H}_{2} \mathrm{O}$ content of the experimental glasses by Raman spectroscopy (Zajacz et al. 2005; Di Muro et al. 2006; Mercier et al. 2008), and compared these results with the theoretical $\mathrm{H}_{2} \mathrm{O}$-content calculated from the initial $\mathrm{H}_{2} \mathrm{O}$ content and the fractions of liquid and amphibole through mass balance calculations (Table 2).

Our results (Table 2) show relatively large differences between calculated and measured $\mathrm{H}_{2} \mathrm{O}$ contents, but with the exception of a few runs, the difference is $<20 \%$, which is acceptable, given the large uncertainties inherent in Raman spectroscopy (see below). We conclude that no significant $\mathrm{H}_{2} \mathrm{O}$ loss has occurred in the reported experiments, with the exception of run $\mathrm{rp} 43\left(1.2 \mathrm{GPa}, 900^{\circ} \mathrm{C}, 8\right.$ $\mathrm{wt} \% \mathrm{H}_{2} \mathrm{O}$ ) that is more consistent with about $5 \mathrm{wt} \% \mathrm{H}_{2} \mathrm{O}$ in the starting material, in line with the $\mathrm{H}_{2} \mathrm{O}$ content determined by Raman spectroscopy. Following Ratajeski and Sisson (1999), which suggest that at high $f_{2}(\mathrm{NNO}+1)$, loss of $\mathrm{Fe}$ to predominantly gold-based alloys is negligible ( $<2 \%$ relative) for a wide range of sample to capsule mass ratios. Wilke and Behrens (1999) concluded that $\mathrm{BN}$ jackets used as a buffer material for water-saturated experiments seems to be appropriate to avoid $\mathrm{H}_{2} \mathrm{O}$ loss, but on the other hand, Sisson et al. (2005) and Kägi et al. (2005) demonstrated that the intrinsic $f_{2}$ buffered by the 'sample-in-sample' double capsule technique is generally 
Table 2 Experimental run conditions, phase assemblage and proportions

\begin{tabular}{|c|c|c|c|c|c|c|c|c|c|c|c|c|c|}
\hline Run no. & $\begin{array}{l}\text { Starting } \\
\text { material }\end{array}$ & $\begin{array}{l}\text { Initial } \\
\mathrm{H}_{2} \mathrm{O} \\
\text { wt\% }\end{array}$ & $\mathrm{T}\left({ }^{\circ} \mathrm{C}\right)$ & $\mathrm{P}(\mathrm{GPa}$ & ime (h) & Capsule technique & Assembly & Run products & Phase proportions wt $\%$ & $\begin{array}{c}\mathrm{H}_{2} \mathrm{O} \\
\text { (melt) }\end{array}$ & $\begin{array}{l}\mathrm{H}_{2} \mathrm{O} \\
\text { calc }\end{array}$ & $\Sigma \mathrm{R}^{2}$ & $\Delta \mathrm{Fe} \%$ \\
\hline $\begin{array}{c}\text { RP30 } \\
\end{array}$ & 8 & 88 & 1000 & 1.2 & 20 & 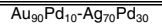 & "NaCl-Pyrex-BN & "liq & $100(21)$ & 7.5 & 8.00 & 0.20 & 0.00 \\
\hline RP31 & 8 & 8 & 950 & 1.2 & 45 & $\mathrm{Au}_{90} \mathrm{Pd}_{10}-\mathrm{Ag}_{70} \mathrm{Pd}_{30}$ & NaCl-Pyrex-BN & liq, grt, amph, ilm & $82.4(17), 12.1(76), 5.3(42), 0.01(5)$ & 9.1 & 9.58 & 0.09 & 3.20 \\
\hline RP43 & 8 & 8 & 900 & 1.2 & 19 & $\mathrm{Au}_{90} \mathrm{Pd}_{10}-\mathrm{Ag}_{70} \mathrm{Pd}_{30}$ & NaCl-Pyrex-BN & liq, grt, amph, plg, ilm & $61.3(3), 11.3(8), 15.4(13), 11.6(3), 0.4(1)$ & 8.7 & 12.55 & 0.00 & -3.70 \\
\hline RP39 & 8 & 8 & 850 & 1.2 & 63 & $\mathrm{Au}_{90} \mathrm{Pd}_{10}-\mathrm{Ag}_{70} \mathrm{Pd}_{30}$ & NaCl-Pyrex-BN & liq, grt, amph, plg & $64.7(21), 12.5(13), 17.9(73), 4.7(59)$ & 11.0 & 11.81 & 0.20 & -0.54 \\
\hline $\begin{array}{l}\text { RP37 } \\
\end{array}$ & 6 & 6 & 1000 & 1.2 & 24 & $\mathrm{Au}_{90} \mathrm{Pd}_{10}-\mathrm{Pt}^{\mathrm{a}}$ & NaCl-Pyrex-BN & liq, grt & $93.1(7), 6.9(10)$ & 5.4 & 6.35 & 0.06 & 15.60 \\
\hline RP90 & $6 c$ & 6 & 950 & 1.2 & 48 & $\mathrm{Au}_{90} \mathrm{Pd}_{10}$ & NaCl-Pyrex-BN & liq, grt, cpx, am & $76.6(21), 22.1(35), 1.1(21)$, trace & 9.0 & 7.83 & 0.32 & 7.60 \\
\hline RP89 & $6 c$ & 6 & 900 & 1.2 & 42 & $\mathrm{Au}_{90} \mathrm{Pd}_{10}$ & NaCl-Pyrex-BN & liq, grt, amph & $76.0(24), 8.4(54), 14.9(64)$ & 11.0 & 7.50 & 0.34 & -6.67 \\
\hline 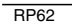 & $4 \mathrm{c}$ & 4 & 1000 & 1.2 & 49 & $\mathrm{Au}_{90} \mathrm{Pd}_{10}-\mathrm{Pt}$ & NaCl-Pyrex-BN & liq, grt, $\mathrm{cpx}$ & $90.8(11), 8.0(15)$, trace & 5.2 & 4.65 & 0.36 & 1.52 \\
\hline RP26 & 4 & 4 & 950 & 1.2 & 49 & $\mathrm{Au}_{90} \mathrm{Pd}_{10}-\mathrm{Ag}_{70} \mathrm{Pd}_{30}$ & NaCl-Pyrex-BN & liq, grt, $\mathrm{cpx}$ & $63.1(32), 35.5(49), 1.2(33)$ & 6.5 & 6.34 & 1.21 & -0.12 \\
\hline RP62A & $4 \mathrm{c}$ & 4 & 900 & 1.2 & 48 & $\mathrm{Au}_{90} \mathrm{Pd}_{10}-\mathrm{Pt}$ & $\mathrm{NaCl}$-Pyrex- $\mathrm{Al}_{2} \mathrm{O}_{3}-\mathrm{BN}-\mathrm{MgO}$ & liq, grt, cpx, plg, opx, ilm & $41.9(32), 13.2(54), 4.9(14), 31.9(51), 7.2(28), 0.8(4)$ & 7.7 & 9.88 & 0.09 & -8.00 \\
\hline Rp88 & $4 \mathrm{c}$ & 4 & 850 & 1.2 & 42 & $\mathrm{Au}_{90} \mathrm{Pd}_{10}$ & NaCl-Pyrex-BN & liq, grt, amph, plg, ilm & $33.3(26), 6.81(44), 27.0(55), 29.8(26),<0.1$ & 9.6 & 9.80 & 0.06 & -6.51 \\
\hline RP66 & $8 \mathrm{c}$ & 8 & 1000 & 0.8 & 48 & $\mathrm{Au}_{90} \mathrm{Pd}_{10}-\mathrm{Pt}$ & $\mathrm{NaCl}-\mathrm{Pyrex}-\mathrm{Al}_{2} \mathrm{O}_{3}-\mathrm{BN}-\mathrm{MgO}$ & liq & $100(4)$ & 5.5 & 8.00 & 1.65 & 13.60 \\
\hline RP32 & 8 & 8 & 950 & 0.8 & 45 & $\mathrm{Au}_{90} \mathrm{Pd}_{10}-\mathrm{Ag}_{70} \mathrm{Pd}_{30}$ & NaCl-Pyrex-BN & liq, amph & $92.9(27), 7.1(57)$ & 7.6 & 8.46 & 0.76 & -5.57 \\
\hline RP22 & 8 & 8 & 900 & 0.8 & 49 & $\mathrm{Au}_{90} \mathrm{Pd}_{10}-\mathrm{Ag}_{70} \mathrm{Pd}_{30}^{\mathrm{a}}$ & NaCl-Pyrex-BN & liq, grt, amph, plg, ilm & $69.4(28), 9.1(18), 13.6(24), 7.5(10), 0.3(9)$ & 11.7 & 11.21 & 0.06 & -0.10 \\
\hline RP25 & 8 & 8 & 850 & 0.8 & 20 & $\mathrm{Au}_{90} \mathrm{Pd}_{10}-\mathrm{Ag}_{70} \mathrm{Pd}_{30} \mathrm{a}^{\mathrm{a}}$ & NaCl-Pyrex-BN & liq, grt, amph, plg, ilm & $66.9(51), 3.8(18), 18.2(10), 10.8(8), 0.1(1)$ & 12.3 & 11.41 & 0.93 & 0.37 \\
\hline $\mathrm{RP} 33$ & 8 & 8 & 800 & 0.8 & 60 & $\mathrm{Au}_{90} \mathrm{Pd}_{10}-\mathrm{Ag}_{70} \mathrm{Pd}_{30}^{\mathrm{a}}$ & NaCl-Pyrex-BN & liq, grt, amph, plg, ilm & $48.7(4), 6.4(14), 22.0(7), 22.2(6), 0.5(1)$ & 15.3 & 15.52 & 0.72 & -1.40 \\
\hline RP18 & 6 & 6 & 950 & 0.8 & 48 & $\mathrm{Au}_{90} \mathrm{Pd}_{10}-\mathrm{Ag}_{70} \mathrm{Pd}_{30}$ & NaCl-Pyrex-BN & liq, grt, amph, plg, ilm & $79.1(23), 1.3(46), 12.4(38), 7.1,(21), 0.01(6)$ & 7.6 & 7.27 & 0.15 & 0.80 \\
\hline RP10 & 6 & 6 & 900 & 0.8 & 48 & $\mathrm{Au}_{90} \mathrm{Pd}_{10}-\mathrm{Ag}_{70} \mathrm{Pd}_{30}^{\mathrm{a}}$ & NaCl-Pyrex-BN & liq, grt, amph, plg, ilm & $67.0(31), 0.1(29), 21.3(59), 11.0,(21), 0.01(6)$ & 9.8 & 8.32 & 0.32 & 1.30 \\
\hline RP34 & 6 & 6 & 850 & 0.8 & 50 & $\mathrm{Au}_{90} \mathrm{Pd}_{10}-\mathrm{Ag}_{70} \mathrm{Pd}_{30}^{\mathrm{a}}$ & NaCl-Pyrex-BN & liq, amph, plg, ilm, grt ${ }^{b}$ & $58.5(20), 26.7(53), 14.4(21), 0.4(8)$ & 10.6 & 9.55 & 0.37 & 0.77 \\
\hline RP13 & 6 & 6 & 800 & 0.8 & 63 & $\mathrm{Au}_{90} \mathrm{Pd}_{10}-\mathrm{Ag}_{70} \mathrm{Pd}_{30}^{\mathrm{a}}$ & NaCl-Pyrex-BN & liq, amph, plg, ilm & $48.6(56), 27.6(10), 23.5(50), 0.2(17)$ & 13.0 & 11.21 & 0.86 & -3.64 \\
\hline RP36 & 6 & 6 & 800 & 0.8 & 60 & $\mathrm{Au}_{90} \mathrm{Pd}_{10}-\mathrm{Ag}_{70} \mathrm{Pd}_{30}^{\mathrm{a}}$ & NaCl-Pyrex-BN & liq, amph, plg, ilm & $45.0(33), 30.6(68), 23.9(25), 0.6(13)$ & 12.2 & 11.97 & 0.96 & 2.95 \\
\hline Rp67 & $4 \mathrm{c}$ & 4 & 1000 & 0.8 & 48 & $\mathrm{Au}_{90} \mathrm{Pd}_{10}-\mathrm{Pt}$ & $\mathrm{NaCl}-$ Pyrex- $-\mathrm{Al}_{2} \mathrm{O}_{3}-\mathrm{BN}-\mathrm{MgO}$ & liq, spl, mag & $97.5(4), 2.4(5),<0.1(3)$ & 4.0 & 4.10 & 0.31 & 5.58 \\
\hline RP14 & 4 & 4 & 950 & 0.8 & 49 & $\mathrm{Au}_{90} \mathrm{Pd}_{10}-\mathrm{Ag}_{70} \mathrm{Pd}_{30}{ }^{\mathrm{a}}$ & NaCl-Pyrex-BN & liq, amph, plg, sple & $74.5(16), 17.4(16), 7.8(11)$, trace & 7.6 & 4.91 & 0.14 & -0.71 \\
\hline RP15 & 4 & 4 & 900 & 0.8 & 49 & $\mathrm{Au}_{90} \mathrm{Pd}_{10}-\mathrm{Ag}_{70} \mathrm{Pd}_{30}{ }^{\mathrm{a}}$ & NaCl-Pyrex-BN & liq, amph, plg, spl, ilm & $68.3(22), 21.7(23), 8.9(18), 0.9(7)$, trace & 9.5 & 5.29 & 0.26 & 0.03 \\
\hline $\mathrm{RP} 40$ & 4 & 4 & 850 & 0.8 & 46 & $\mathrm{Au}_{90} \mathrm{Pd}_{10}-\mathrm{Ag}_{70} \mathrm{Pd}_{30}$ & NaCl-Pyrex-BN & liq, amph, plg, ilm & $53.6(19), 25.0(28), 20.6(19), 0.9(8)$ & 10.0 & 6.53 & 0.68 & -1.16 \\
\hline
\end{tabular}

Values in Italics indicate $>20 \%$ difference between theoretical and measured $\mathrm{H}_{2} \mathrm{O}$ content as determined Raman spectroscopy

$\Delta \mathrm{Fe} \%$ apparent gain or loss of iron, calculated as: $100 \times$ (total $\mathrm{Fe}_{\text {calc }}-$ total $\mathrm{FeO}$ in the starting material)/total $\mathrm{FeO}$ in the starting material. Positive values: iron gain, negative values, iron loss; $\mathrm{H}_{2} \mathrm{O}$ (melt) $\mathrm{H}_{2} \mathrm{O}$ content determined by Raman spectroscopy, applying the measurement protocol of Di Muro et al. (2006), and Mercier et al. (2008); $\mathrm{H}_{2} \mathrm{O}$ calc theroretical $\mathrm{H}_{2} \mathrm{O}$ content of the silicate liquid determined by the starting material and the appropriate proportions of $\mathrm{H}_{2} \mathrm{O}$ incorporated into hydrous minerals

${ }^{a}$ Fe-presaturated inner Au-Pd capsule, following techniques described by Kägi et al. (2005)

${ }^{\mathrm{b}}$ Garnet is present but was not used for mass balance (negative)

c Spinel is present, but too small for analysis, spinel from RP15 was therefore used for the mass balance

overcome by the buffering capacity of $\mathrm{BN}$. In order to avoid $\mathrm{H}_{2} \mathrm{O}$ loss, we used the piston-cylinder assemblies similar to those of Kägi et al. (2005); Fig. 2b, BN setup). Experimental conditions ranged between 800 and $1,000^{\circ} \mathrm{C}$ and $0.8-1.2 \mathrm{GPa}$ and run durations varied from 24 to $60 \mathrm{~h}$ (Table 2). Most experiments conducted in this study employing the double capsule technique have been performed with a combination of $\mathrm{Au}_{90} \mathrm{Pd}_{10}$ (inner) and $\mathrm{Pt}$ or $\mathrm{Ag}_{70} \mathrm{Pd}_{30}$ (outer) capsules. Duplicate experiments at identical pressure-temperature- $\mathrm{X}_{\mathrm{H}_{2} \mathrm{O}}$ conditions (e.g. rp13 and rp36; see Table 2), in general, resulted in very similar melt fractions, phase assemblages and phase compositions indicating good reproducibility of the results. Previous experimental studies on tonalite or andesite bulk compositions with $\mathrm{H}_{2} \mathrm{O}$ and $\mathrm{H}_{2} \mathrm{O}-\mathrm{CO}_{2}$ mixtures were often performed in $\mathrm{Ag}_{70} \mathrm{Pd}_{30}$ capsules in the range of 1-3 GPa (Allen and Boettcher 1978, 1983; Allen et al. 1975; Green 1972; Green and Ringwood 1968a; Huang and Wyllie 1986; Stern et al. 1975). Due to the choice of single capsule non-pretreated alloys as capsule material, the results of these earlier studies, however, must be carefully evaluated for $\mathrm{H}_{2} \mathrm{O}$ loss and/or oxidation problems, before they can directly be compared with our new experiments.

Recovered capsules were mounted in epoxy resin, ground with sandpaper to expose a longitudinal cross- section of the charge, and polished with diamond paste of different grades. After carbon coating, major element compositions of experimental liquids and solid phases were determined with a Cameca SX50 and a JEOL 8600 electron microprobe analyzer at ETH Zürich. We employed an accelerating voltage of $15 \mathrm{kV}$ and a beam current of $20 \mathrm{nA}$, using silicates and oxides as standards. Analytical errors associated with $\mathrm{Na}$ loss in hydrous $\mathrm{SiO}_{2}$-rich glasses during microprobe analysis have been minimized by reducing the probe current to $7 \mathrm{nA}$, increasing the spot size to $10 \mu \mathrm{m}$ and limiting the counting time to $3 \mathrm{~s}$ for $\mathrm{Na}$. Results on experimental glasses and minerals are listed in Table 3.

Bulk $\mathrm{H}_{2} \mathrm{O}$ and $\mathrm{CO}_{2}$ contents of some of the starting materials were determined by Fourier transform infrared spectroscopy (FT-IR) at Caltech on rapidly fused starting material at high pressures (Table 4). Powders were packed into single $\mathrm{Au}_{90} \mathrm{Pd}_{10}$ capsules, redried overnight at $110^{\circ} \mathrm{C}$ and then sealed by arc welding. Samples were hold at 1.6 GPa and $1,150^{\circ} \mathrm{C}$, for $30 \mathrm{~min}$, yielding crystal and bubble-free glasses. Absorption spectra were measured on glass wafers prepared from the center of the capsules. Concentrations were determined through the Beer-Lambert law with absorbance from composition-dependent expressions for the $1,430 \mathrm{~cm}^{-1}$ absorption band for $\mathrm{CO}_{3}{ }^{2-}$, and for the 3,500 and $5,200 \mathrm{~cm}^{-1}$ for $\mathrm{H}_{2} \mathrm{O}$. Resulting bulk 

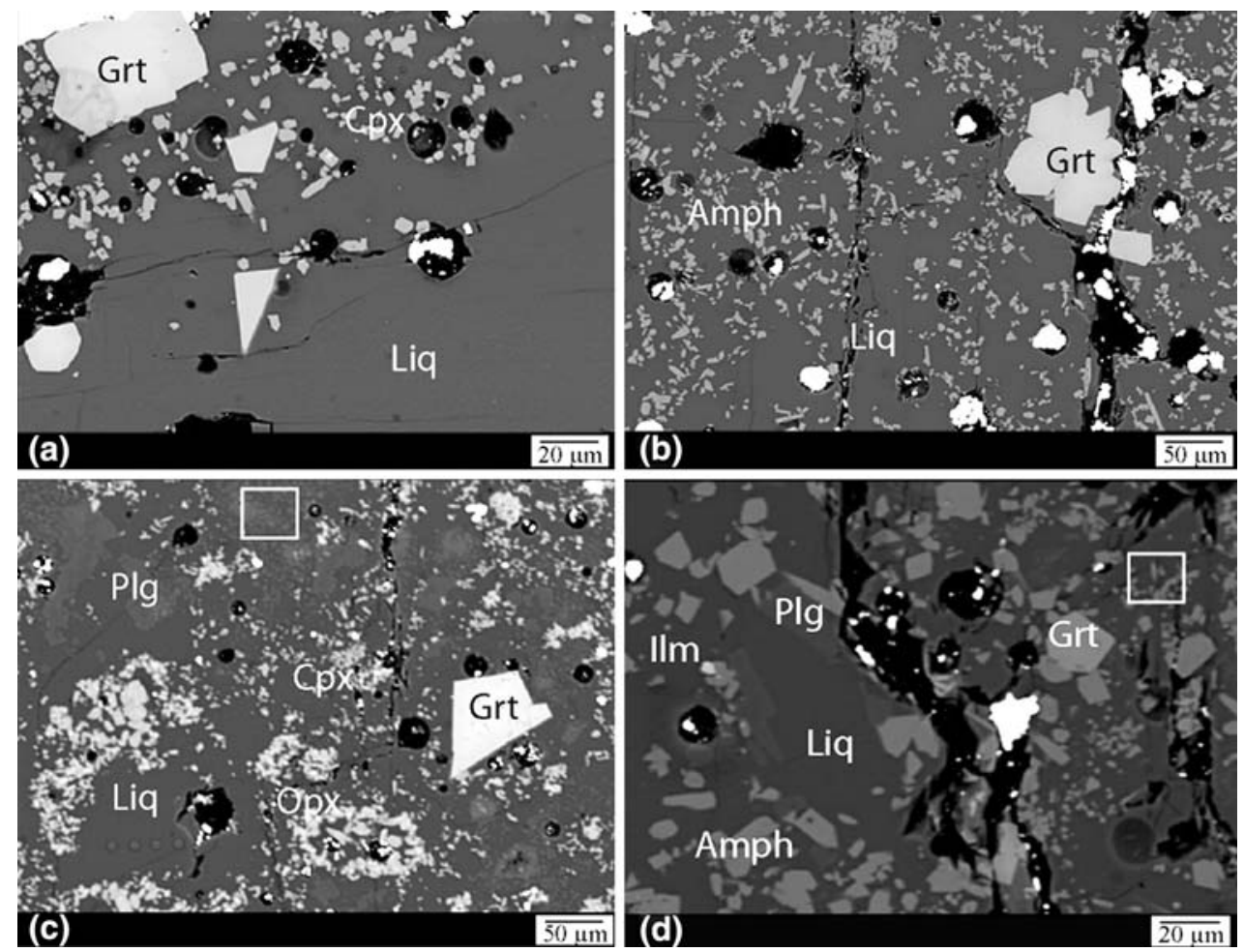

Fig. 2 Backscatter electron images (BSE) of run products. Phases identified in the experiments include garnet (Grt), high-Ca pyroxene (Cpx), low-Ca pyroxene (Opx), amphibole (Amph), plagioclase (Plg), ilmenite (Ilm) and melt (Liq). a Run RP90 (garnet + cpx + liquid), $1.2 \mathrm{GPa}, 6 \mathrm{wt} \% \mathrm{H}_{2} \mathrm{O}$ and $950^{\circ} \mathrm{C}$; b Run RP89, (garnet + amphibole + liquid), $1.2 \mathrm{GPa}, 6 \mathrm{wt} \% \mathrm{H}_{2} \mathrm{O}$ and $900^{\circ} \mathrm{C}$; c Run RP62A (garnet + clinopyroxene + orthopyroxene + plagioclase + liquid) at $1.2 \mathrm{GPa}, 6 \mathrm{wt} \% \mathrm{H}_{2} \mathrm{O}$ and $950^{\circ} \mathrm{C} ; \quad$ d Run $\mathrm{RP} 18$

(garnet + amphibole + plagioclase + liquid) at $0.8 \mathrm{GPa}, 6 \mathrm{wt} \%$ $\mathrm{H}_{2} \mathrm{O}$ and $950^{\circ} \mathrm{C}$; The boxes highlight needle-like crystal and subhedral quench plagioclases. The majority of the experiments contain traces of ilmenite and/or spinel. Scale bar is $20-50 \mu \mathrm{m}$. Note the overall small grain size (except for garnet). Note also that abundant bubbles $(\sim 5$ to $>10 \mu \mathrm{m})$ can be observed, indicating vapor saturation of most of the experiments

water and $\mathrm{CO}_{2}$ contents were determined following the procedures of Dixon et al. (1995) for $\mathrm{H}_{2} \mathrm{O}$ and Pilet et al. (2008) for $\mathrm{CO}_{2}$ and are listed in Table 4. Resulting $\mathrm{CO}_{2}$ contents $(\sim 0.9 \mathrm{wt} \%)$ are higher than expected for our starting material, but consistent with the observation that many experimental runs contain small bubbles indicating saturation with a fluid phase. However, the $\mathrm{CO}_{2}$ concentrations are likely to be lower for the andesite experiments at 0.8 and $1.2 \mathrm{GPa}$, because the experiments on the starting material were run after the phase equilibrium experiments were terminated. Since we used synthetic starting materials, there are several potential sources of $\mathrm{CO}_{2}$, such as incomplete degassing of $\mathrm{CaCO}_{3}$ during preparation of the starting material, traces of hydrocarbon left from the denatured ethanol during grinding (see also Sisson et al. 2005), or decarbonated $\mathrm{CaO}$ progressively recarbonates by fixing $\mathrm{CO}_{2}$ from the air.

The $\mathrm{H}_{2} \mathrm{O}$ contents of all experiments were determined by Micro-Raman spectroscopy. Raman scattering was excited using a $488 \mathrm{~nm} \mathrm{Ar}+$ Laser (Inova 90) and measurements were performed with a LabRam II Micro-Raman

spectrometer at ETH Zurich. Glasses were analyzed by focusing the laser beam on the sample surface, with $\sim 1$ $2 \mathrm{~mm}$ spotsize under an Olympus microscope in confocal mode. The instrument is equipped with a beam splitter that allows $70 \%$ of the scattered light to reach the detector after passing through a notch filter. Spectra were obtained in the $200-1,500$ and $2,800-3,900 \mathrm{~cm}^{-1}$ ranges to cover low and high frequency $\mathrm{T}-\mathrm{O}$ stretching and vibration modes and the $\mathrm{OH} / \mathrm{H}_{2} \mathrm{O}$ stretching regions. Average input laser power was about $600 \mathrm{~mW}$. Spectra acquisition times were $120 \mathrm{~s}$; three measurements were conducted on each spot and three different spots were analyzed on each sample. We did not apply Long-correction to our raw data as this does not improve the water calibration (see Mercier et al. 2008) and performed a cubic baseline correction scheme along the strategies described by Di Muro et al. (2006) and Mercier et al. (2008). Calibration was conducted with a series of internal standard glasses ranging from basalts to andesites to rhyolites and $\mathrm{H}_{2} \mathrm{O}$ contents from 0.0 to $11.0 \mathrm{wt} \%$ that have been measured by FTIR, SIMS and/or Karl-Fisher titration (KFT). The calibration bases on the evaluation of 
Table 3 Electron microprope analyses of run products, with all $\mathrm{Fe}$ as $\mathrm{FeO}$

\begin{tabular}{|c|c|c|c|c|c|c|c|c|c|c|c|c|c|}
\hline Run no. & Phase & $n$ & $\mathrm{SiO}_{2}$ & $\mathrm{TiO}_{2}$ & $\mathrm{Al}_{2} \mathrm{O} 3$ & $\mathrm{FeO}_{\text {tot }}$ & $\mathrm{MnO}$ & $\mathrm{MgO}$ & $\mathrm{CaO}$ & $\mathrm{Na}_{2} \mathrm{O}$ & $\mathrm{K}_{2} \mathrm{O}$ & Total & $\mathrm{Mg} \#$ \\
\hline P30 & $\mathrm{gl}$ & 11 & $53.0(8)$ & 0.69 (5) & $15.5(5)$ & $6.2(4)$ & $.26(8)$ & $2.35(12)$ & $6.68(28)$ & $2.33(17)$ & $1.23(9)$ & 88.3 & 0.403 \\
\hline \multirow[t]{4}{*}{ RP31 } & $\mathrm{gl}$ & 18 & $55.3(8)$ & 7) & 15.4 (2) & $5.91(18)$ & $.22(5)$ & 1 & $6.04(17)$ & $2.88(16)$ & $1.45(8)$ & 89.5 & 0.325 \\
\hline & grt & 15 & $38.3(4)$ & 1.07 (9) & 20.1 (2) & $21.6(12)$ & $2.14(7)$ & $8.02(26)$ & $9.08(33)$ & $0.04(2)$ & 0.01 (1) & 100.4 & 0.398 \\
\hline & amph & 16 & $41.0(7)$ & $1.51(25)$ & $15.1(6)$ & $13.8(9)$ & 0.36 (3) & $11.6(8)$ & $10.8(3)$ & $2.13(8)$ & 0.78 (3) & 97.0 & 0.601 \\
\hline & ilm & 6 & 0.03 & $40.4(18)$ & 0.69 (6) & $53.0(26)$ & $0.52(8)$ & 2.33 (19) & 0.08 & 0.01 (1) & $0.01(0)$ & 96.7 & 0.068 \\
\hline \multirow[t]{5}{*}{ RP43 } & $\mathrm{gl}$ & 12 & $61.6(6)$ & $0.50(7)$ & $16.4(3)$ & $3.26(18)$ & $0.06(5)$ & $0.82(6)$ & $4.87(12)$ & $3.08(22)$ & $1.90(8)$ & 92.5 & 0.309 \\
\hline & grt & 8 & $38.3(2)$ & $1.12(10)$ & $20.3(2)$ & $23.3(6)$ & $1.54(21)$ & $6.81(29)$ & $9.13(45)$ & 0.02 (1) & 0.01 (1) & 100.5 & 0.343 \\
\hline & amph & 16 & 40.7 (7) & $1.65(33)$ & $15.0(4)$ & $16.5(6)$ & 0.25 (4) & $10.1(6)$ & $10.5(3)$ & $2.08(7)$ & $0.70(7)$ & 97.4 & 0.521 \\
\hline & plg & 4 & $52.3(2)$ & 0.08 & 29.4 (1) & 0.35 & 0.01 (1) & 0.07 (4) & 12.7 (4) & $4.02(18)$ & $0.24(0)$ & 100.3 & 0.082 \\
\hline & ilm & 5 & $0.11(10)$ & 43.7 (17) & $0.73(8)$ & $50.4(14)$ & 0.21 (3) & $2.32(13)$ & $0.18(8)$ & 0.01 (1) & 0.02 & 97.7 & 0.076 \\
\hline \multirow[t]{4}{*}{ RP39 } & $\mathrm{gl}$ & 10 & $60.3(7)$ & $0.30(6)$ & 16.5 (4) & $2.31(23)$ & $0.06(5)$ & $0.54(6)$ & $5.02(20)$ & 2.88 (19) & $1.67(6)$ & 89.5 & 0.294 \\
\hline & grt & 11 & $37.9(6)$ & $1.24(14)$ & 20.1 (3) & $23.2(15)$ & $1.55(26)$ & $4.39(35)$ & 11.7 (4) & 0.05 (2) & 0.02 (2) & 100.1 & 0.252 \\
\hline & amph & 7 & $40.5(9)$ & $1.31(11)$ & $15.2(6)$ & $16.8(6)$ & $0.35(71)$ & $9.4(6)$ & 10.9 (3) & $2.22(8)$ & $0.82(5)$ & 97.4 & 0.500 \\
\hline & plg & 7 & 49.4 (7) & $0.06(5)$ & $31.8(8)$ & $0.74(29)$ & 0.02 & 0.08 (5) & $15.1(7)$ & $2.33(25)$ & $0.19(6)$ & 99.8 & 0.159 \\
\hline \multirow[t]{2}{*}{ RP37 } & $\mathrm{gl}$ & 26 & $53.0(7)$ & $0.83(6)$ & $16.8(3)$ & $7.8(4)$ & $0.26(6)$ & $2.38(11)$ & $6.57(18)$ & $2.95(16)$ & $1.43(6)$ & 92.1 & 0.352 \\
\hline & grt & 21 & $39.5(5)$ & 0.79 (39) & 21.5 & $19.5(8)$ & $1.60(21)$ & $9.45(66)$ & $7.72(80)$ & 0.02 (2) & 0.02 (2) & 100.4 & 0.46 \\
\hline \multirow[t]{3}{*}{ RP90 } & $\mathrm{gl}$ & 23 & 56.7 (3) & 0.75 (4) & $16.5(2)$ & $4.01(10)$ & 0.11 (3) & $1.20(5)$ & $5.52(13)$ & $2.62(6)$ & $1.60(4)$ & 89.0 & 0.349 \\
\hline & grt & 14 & 38.6 (3) & $1.46(14)$ & $20.2(2)$ & $20.7(4)$ & $1.44(25)$ & $7.28(39)$ & $9.70(37)$ & 0.03 (2) & 0.01 (1) & 99.4 & 0.385 \\
\hline & $\operatorname{cpx}$ & 7 & 46.9 (3) & $1.07(5)$ & $9.14(24)$ & $10.7(4)$ & $0.30(8)$ & 10.0 & $20.3(3)$ & 0.59 (3) & 0.02 (1) & 99.0 & 0.627 \\
\hline \multirow[t]{3}{*}{ RP89 } & $\mathrm{gl}$ & 18 & 54.8 & 0.57 (4) & $16.4(2)$ & $3.23(11)$ & 0.14 (3) & 0.77 (4) & $5.55(10)$ & $2.20(38)$ & $1.43(5)$ & 85.1 & 0.298 \\
\hline & grt & 19 & 37.5 (4) & $1.45(7)$ & 20.4 (2) & 21.5 & $2.04(26)$ & $5.55(20)$ & $10.2(4)$ & 0.05 (2) & $0.01(1)$ & 98.7 & 0.315 \\
\hline & amph & 7 & $40.0(6)$ & $1.85(10)$ & $16.0(8)$ & $15.5(5)$ & 0.37 (3) & $9.48(47)$ & $10.6(2)$ & $1.90(6)$ & $0.82(4)$ & 96.5 & 0.522 \\
\hline \multirow[t]{3}{*}{ RP62 } & $\mathrm{gl}$ & 10 & $55.4(3)$ & 0.87 (2) & $16.6(1)$ & $6.91(14)$ & 0.17 (2) & $1.93(6)$ & $6.67(6)$ & $2.58(7)$ & $1.52(3)$ & 92.6 & 0.332 \\
\hline & grt & 5 & 39.0 (4) & $1.12(25)$ & 20.7 (3) & 19.9 (1) & $1.36(14)$ & $9.33(24)$ & $7.98(38)$ & 0.03 (3) & $<0.01$ & 99.4 & 0.45 \\
\hline & cpx & 7 & $48.3(8)$ & $0.71(9)$ & $7.8(7)$ & $11.2(8)$ & $0.40(6)$ & $12.2(6)$ & $19.2(3)$ & $0.60(2)$ & 0.04 (2) & 99.0 & 0.639 \\
\hline \multirow[t]{3}{*}{ RP26 } & $\mathrm{gl}$ & 13 & $61.0(3)$ & 0.75 (3) & $15.6(1)$ & $4.29(6)$ & 0.09 (2) & 1.03 (3) & $5.07(5)$ & $2.90(22)$ & $1.85(2)$ & 92.5 & 0.300 \\
\hline & grt & 5 & 38.5 (3) & $1.50(21)$ & 20.2 (3) & $23.5(8)$ & $1.55(11)$ & $7.4(8)$ & $8.05(34)$ & 0.04 (2) & $<0.01$ & 100.7 & 0.359 \\
\hline & $\mathrm{cpx}$ & 4 & $48.1(8)$ & 0.85 (3) & $8.32(60)$ & $12.3(6)$ & 0.29 (9) & $10.5(3)$ & $18.5(7)$ & $0.71(8)$ & $<0.01$ & 99.6 & 0.60 \\
\hline \multirow[t]{6}{*}{ RP62A } & $\mathrm{gl}$ & 25 & $62.8(11)$ & $0.45(5)$ & 14.7 (7) & $2.84(56)$ & 0.05 (4) & $0.90(57)$ & $4.12(92)$ & $2.46(25)$ & $2.32(13)$ & 90.6 & 0.362 \\
\hline & grt & 28 & $38.3(8)$ & $1.50(20)$ & 20.3 (9) & $24.5(11)$ & $1.46(46)$ & $6.11(86)$ & $8.40(50)$ & 0.05 (3) & $<0.01$ & 100.7 & 0.308 \\
\hline & $\mathrm{cpx}$ & 12 & $48.8(10)$ & $0.64(10)$ & $6.53(56)$ & $16.2(15)$ & $0.50(10)$ & $10.1(8)$ & $16.4(16)$ & $0.64(11)$ & 0.07 (5) & 100.0 & 0.526 \\
\hline & plg & 19 & $55.5(10)$ & 0.04 (3) & 27.1 (9) & $0.52(38)$ & $<0.02$ & $<0.04$ & $10.6(8)$ & $4.74(31)$ & $0.36(7)$ & 98.9 & 0.175 \\
\hline & opx & 6 & $48.6(8)$ & 0.31 (3) & $5.90(98)$ & $26.4(8)$ & $0.72(10)$ & 15.7 (9) & 1.79 (27) & 0.07 (4) & (3) & 99.6 & 0.513 \\
\hline & ilm & 9 & $0.44(11)$ & $48.6(10)$ & 0.51 (4) & $43.0(2)$ & $0.31(0)$ & $3.6(2)$ & 0.2 & 0.01 (1) & 0) & 96.9 & 0.131 \\
\hline \multirow[t]{4}{*}{ RP88 } & $\mathrm{gl}$ & 18 & $65.8(7)$ & $0.23(3)$ & 13.7 (2) & $1.94(11)$ & 0.09 (4) & 0.26 & $3.30(10)$ & $1.01(46)$ & $1.98(12)$ & 88.3 & 0.196 \\
\hline & grt & 17 & $37.6(8)$ & $1.32(18)$ & $19.2(10)$ & $22.9(6)$ & $3.21(51)$ & $3.76(40)$ & $10.5(7)$ & 0.07 (6) & $<0.01$ & 98.5 & 0.226 \\
\hline & amph & 19 & $41.4(5)$ & 1.67 (17) & $13.6(6)$ & $18.1(7)$ & 0.49 (6) & $8.48(38)$ & $10.4(3)$ & $1.73(7)$ & 0.69 (5) & 96.6 & 0.455 \\
\hline & plg & 9 & $55.6(10)$ & 0.03 (2) & $26.4(7)$ & $0.39(10)$ & 0.02 (2) & $<0.03$ & $9.66(89)$ & $5.21(36)$ & $0.31(5)$ & 97.7 & 0.000 \\
\hline P66 & $\mathrm{gl}$ & 15 & $52.6(2)$ & $0.86(5)$ & $17.2(2)$ & 8.23 (16) & 0.38 (4) & $2.78(7)$ & $7.25(9)$ & $2.55(9)$ & $1.32(5)$ & 93.2 & 0.376 \\
\hline \multirow[t]{2}{*}{ RP32 } & gl & 11 & $54.0(4)$ & $0.84(8)$ & 16.1 (2) & $6.20(36)$ & $0.26(9)$ & $2.03(12)$ & $6.26(16)$ & $2.92(12)$ & 1.47 (5) & 90.2 & 0.368 \\
\hline & amph & 7 & 42.5 (4) & 2.92 (4) & $12.5(2)$ & $12.5(3)$ & $0.40(2)$ & $13.2(2)$ & $10.5(2)$ & $2.24(3)$ & $0.51(4)$ & 97.1 & 0.653 \\
\hline \multirow[t]{5}{*}{ RP22 } & $\mathrm{gl}$ & 5 & 59.4 (1) & $0.46(7)$ & $16.3(3)$ & $3.62(11)$ & $0.16(4)$ & $1.00(9)$ & $5.37(21)$ & $2.30(10)$ & $1.62(18)$ & 90.3 & 0.331 \\
\hline & grt & 5 & 39.0 (2) & 0.87 (1) & 21.1 (2) & $24.4(8)$ & $2.62(27)$ & $5.88(29)$ & $7.53(51)$ & 0.03 (2) & $<0.01$ & 101.4 & 0.300 \\
\hline & amph & 3 & 42.1 (3) & $1.91(21)$ & 13.9 (5) & $17.3(4)$ & 0.39 (5) & $10.4(3)$ & 10.1 & 1.84 (1) & 0.58 (4) & 98.6 & 0.521 \\
\hline & $\mathrm{pl}$ & 22 & $47.4(6)$ & 0.05 (3) & 33.9 (6) & $0.40(13)$ & 0.03 (2) & 0.09 (1) & $17.3(8)$ & 1.39 (29) & $0.12(5)$ & 100.9 & 0.203 \\
\hline & ilm & 5 & $0.13(7)$ & $51.9(3)$ & $0.24(1)$ & $42.2(5)$ & $1.35(32)$ & 3.08 (2) & $0.20(4)$ & $<0.02$ & $<0.01$ & 99.0 & 0.116 \\
\hline
\end{tabular}


Table 3 continued

\begin{tabular}{|c|c|c|c|c|c|c|c|c|c|c|c|c|c|}
\hline Run no. & Phase & $n$ & $\mathrm{SiO}_{2}$ & $\mathrm{TiO}_{2}$ & $\mathrm{Al}_{2} \mathrm{O} 3$ & $\mathrm{FeO}_{\text {tot }}$ & $\mathrm{MnO}$ & $\mathrm{MgO}$ & $\mathrm{CaO}$ & $\mathrm{Na}_{2} \mathrm{O}$ & $\mathrm{K}_{2} \mathrm{O}$ & Total & $\mathrm{Mg} \#$ \\
\hline \multirow[t]{5}{*}{ RP25 } & $\mathrm{gl}$ & 7 & $59.7(6)$ & $0.40(15)$ & $16.0(4)$ & 4.37 (326) & $0.22(5)$ & $0.75(10)$ & $5.23(22)$ & $2.36(16)$ & $1.84(24)$ & 90.9 & 0.233 \\
\hline & grt & 3 & $37.6(2)$ & 0.79 (1) & $20.2(1)$ & $25.5(1)$ & $2.56(17)$ & $4.15(05)$ & $8.91(7)$ & $0.02(01)$ & $<0.01$ & 99.8 & 0.225 \\
\hline & amph & 9 & $42.1(9)$ & $1.56(27)$ & $14.1(10)$ & $18.3(7)$ & $0.42(5)$ & $8.97(61)$ & $10.3(5)$ & 1.85 (13) & 0.63 (3) & 98.3 & 0.466 \\
\hline & $\mathrm{pl}$ & 4 & $48.2(6)$ & 0.03 (1) & $32.4(6)$ & $0.56(11)$ & 0.04 (1) & 0.03 (2) & $16.8(3)$ & $1.93(12)$ & $0.13(5)$ & 100.2 & 0.094 \\
\hline & ilm & 5 & $0.21(12)$ & $50.9(6)$ & $0.26(4)$ & $44.0(6)$ & $1.23(5)$ & 1.95 (1) & 0.17 (5) & $<0.01$ & $<0.01$ & 98.7 & 0.073 \\
\hline \multirow[t]{5}{*}{ RP33 } & $\mathrm{gl}$ & 6 & $67.6(9)$ & 0.14 (2) & $13.6(3)$ & 2.18 (218) & $0.10(5)$ & $0.37(6)$ & $2.66(216)$ & $2.64(20)$ & $2.37(10)$ & 91.7 & 0.232 \\
\hline & grt & 8 & 37.4 (2) & $1.03(8)$ & $19.6(2)$ & 27.9 (13) & $2.86(22)$ & $3.60(24)$ & $8.16(71)$ & $0.04(02)$ & 0.02 (1) & 100.6 & 0.187 \\
\hline & amph & 9 & $43.1(9)$ & $1.12(21)$ & $12.8(8)$ & $18.5(14)$ & 0.45 (5) & $8.76(78)$ & $10.3(4)$ & $1.61(8)$ & $0.63(6)$ & 97.2 & 0.458 \\
\hline & $\mathrm{pl}$ & 6 & $47.5(10)$ & 0.04 (2) & $33.5(7)$ & $1.15(31)$ & 0.04 (3) & 0.07 (2) & $15.7(5)$ & $1.73(20)$ & $0.18(6)$ & 99.9 & 0.094 \\
\hline & ilm & 3 & $0.55(27)$ & 48.7 (2) & $0.23(2)$ & $46.0(2)$ & 0.79 (1) & $1.13(5)$ & 0.16 & 0.05 (2) & 0.04 (2) & 97.6 & 0.042 \\
\hline \multirow[t]{5}{*}{ RP18 } & $\mathrm{gl}$ & 26 & $55.7(7)$ & 0.68 (4) & $16.6(2)$ & $6.00(46)$ & $0.30(2)$ & 1.57 (13) & $5.80(18)$ & $2.55(9)$ & $1.51(7)$ & 90.7 & 0.318 \\
\hline & grt & 5 & $37.6(8)$ & $1.32(18)$ & $19.2(10)$ & $22.9(6)$ & $3.21(51)$ & $3.76(40)$ & $10.5(7)$ & 0.07 (6) & $<0.01$ & 98.5 & 0.226 \\
\hline & amph & 5 & $41.4(5)$ & 1.97 (35) & 14.1 (3) & 16.9 (14) & $0.41(5)$ & $10.1(9)$ & $10.0(3)$ & 2.09 (9) & 0.57 (4) & 97.4 & 0.515 \\
\hline & $\mathrm{pl}$ & 6 & $48.3(10)$ & $0.05(0)$ & 31.9 (5) & $0.82(23)$ & 0.04 (1) & $0.18(6)$ & $16.1(7)$ & $1.93(30)$ & $0.16(2)$ & 99.5 & 0.284 \\
\hline & ilm & 2 & $0.40(24)$ & $50.5(2)$ & $0.34(2)$ & $42.6(2)$ & $1.13(1)$ & $3.32(3)$ & $0.45(6)$ & $<0.02$ & $<0.01$ & 98.8 & 0.122 \\
\hline \multirow[t]{5}{*}{ RP10 } & $\mathrm{gl}$ & 7 & $57.9(6)$ & $0.43(2)$ & 15.9 (1) & $4.91(7)$ & $0.21(2)$ & 0.79 (4) & $4.71(11)$ & $2.46(12)$ & $1.80(3)$ & 89.1 & 0.223 \\
\hline & grt & 7 & 37.4 (4) & $0.90(10)$ & $20.3(3)$ & $24.9(11)$ & $2.78(10)$ & $4.86(16)$ & $8.45(26)$ & 0.03 (1) & $<0.01$ & 99.6 & 0.258 \\
\hline & amph & 15 & $41.5(4)$ & 2.24 (19) & $13.1(4)$ & $19.1(8)$ & $0.53(8)$ & $9.14(16)$ & $9.82(23)$ & $2.12(6)$ & $0.60(5)$ & 98.1 & 0.460 \\
\hline & $\mathrm{pl}$ & 8 & $44.6(5)$ & 0.02 (2) & 34.9 (7) & $0.75(54)$ & 0.04 (2) & 0.06 (4) & 17.8 & 1.32 (17) & 0.07 (1) & 99.6 & 0.129 \\
\hline & ilm & 3 & $0.13(10)$ & $51.1(4)$ & 0.27 (4) & $44.0(3)$ & $1.03(3)$ & $2.01(2)$ & $0.13(5)$ & 0.07 (5) & $<0.01$ & 98.3 & 0.075 \\
\hline \multirow[t]{5}{*}{ RP34 } & $\mathrm{gl}$ & 11 & $62.6(5)$ & 0.26 (4) & $16.3(3)$ & 3.62 (17) & $0.15(6)$ & $0.51(8)$ & $4.20(33)$ & $2.72(27)$ & $2.63(28)$ & 92.9 & 0.189 \\
\hline & grt & 6 & 37.9 (3) & $0.95(5)$ & $20.3(2)$ & $25.1(5)$ & $3.10(20)$ & 3.79 (16) & 8.77 (44) & 0.02 (1) & $<0.01$ & 99.9 & 0.212 \\
\hline & amph & 9 & 40.7 (4) & $1.46(25)$ & $14.2(4)$ & 19.9 (5) & 0.48 & $7.70(24)$ & $9.94(35)$ & $2.12(11)$ & $0.61(4)$ & 97.1 & 0.377 \\
\hline & $\mathrm{pl}$ & 6 & $48.4(8)$ & 0.03 (1) & $32.6(9)$ & $0.71(62)$ & $0.03(2)$ & 0.04 (2) & $15.6(7)$ & 2.17 (28) & 0.13 (3) & 99.7 & 0.084 \\
\hline & ilm & 4 & $0.13(5)$ & $50.1(2)$ & $0.25(3)$ & 44.9 (4) & $0.96(1)$ & $1.46(4)$ & $0.21(9)$ & 0.03 (1) & $<0.01$ & 98.0 & 0.054 \\
\hline \multirow[t]{4}{*}{ RP13 } & $\mathrm{gl}$ & 10 & $63.4(1)$ & $0.12(2)$ & $13.8(7)$ & $1.86(8)$ & 0.13 & 0.27 (4) & $2.50(5)$ & $2.16(3)$ & 2.43 (1) & 86.6 & 0.205 \\
\hline & amph & 14 & $43.1(7)$ & $1.22(32)$ & $11.8(7)$ & $22.3(13)$ & $0.54(3)$ & $6.84(69)$ & 9.9 (3) & $1.65(8)$ & $0.61(7)$ & 97.9 & 0.353 \\
\hline & $\mathrm{pl}$ & 7 & 47.9 (13) & $0.02(2)$ & 33.8 (12) & $0.65(40)$ & $0.03(2)$ & 0.07 (2) & $15.9(6)$ & $2.25(31)$ & 0.10 (2) & 100.7 & 0.156 \\
\hline & ilm & 3 & $0.40(14)$ & $51.7(10)$ & 0.19 (6) & 44.4 (4) & $1.28(1)$ & 1.02 (3) & 0.13 & 0.04 (3) & $<0.01$ & 99.2 & 0.039 \\
\hline \multirow[t]{4}{*}{ RP36 } & $\mathrm{gl}$ & 12 & $69.7(6)$ & 0.12 (1) & $13.1(1)$ & $1.75(1)$ & $0.12(8)$ & 0.17 (11) & $1.88(1)$ & $1.94(50)$ & $3.62(1)$ & 92.4 & 0.148 \\
\hline & amph & 9 & $43.5(7)$ & $1.42(8)$ & $13.1(2)$ & $19.5(5)$ & $0.66(10)$ & 8.27 (66) & $9.66(39)$ & $1.84(5)$ & $0.59(7)$ & 98.4 & 0.431 \\
\hline & $\mathrm{pl}$ & 2 & $44.3(2)$ & 0.07 (1) & 35.0 (1) & $3.25(16)$ & 0.11 & 0.24 (1) & $15.9(2)$ & $1.61(5)$ & 0.08 & 100.5 & 0.116 \\
\hline & ilm & 2 & $1.21(39)$ & $53.5(38)$ & $0.28(8)$ & $41.3(20)$ & $1.28(1)$ & $1.24(10)$ & $0.06(2)$ & 0.10 (1) & 0.07 (1) & 99.0 & 0.051 \\
\hline \multirow[t]{3}{*}{ RP67 } & $\mathrm{gl}$ & 15 & $53.5(13)$ & $0.79(21)$ & 16.4 (3) & 7.19 (13) & $0.33(5)$ & $2.86(9)$ & $7.08(11)$ & $2.46(11)$ & $1.45(6)$ & 92.1 & 0.414 \\
\hline & spl & 2 & 0.04 (1) & $0.29(1)$ & $51.0(1)$ & 33.9 (3) & $0.56(4)$ & $13.17(2)$ & 0.17 (2) & $<0.01$ & $<0.01$ & 99.1 & 0.410 \\
\hline & mag & 13 & 0.15 (4) & $4.52(6)$ & $8.02(9)$ & 77.4 (4) & $0.60(2)$ & 3.68 (5) & $0.14(9)$ & $<0.01$ & $<0.01$ & 94.6 & 0.078 \\
\hline \multirow[t]{3}{*}{ RP14 } & $\mathrm{gl}$ & 6 & 54.7 (4) & 0.57 (3) & $16.41(1)$ & $5.88(4)$ & $0.25(4)$ & $1.25(3)$ & $5.08(9)$ & $2.46(11)$ & $1.82(3)$ & 88.4 & 0.274 \\
\hline & amph & 19 & $42.3(8)$ & $2.42(29)$ & $14.3(4)$ & $14.7(5)$ & $0.37(4)$ & 10.9 (5) & $10.4(3)$ & 2.19 (9) & 0.48 (12) & 98.0 & 0.569 \\
\hline & $\mathrm{pl}$ & 6 & $48.8(5)$ & 0.08 (2) & 31.7 (7) & $1.13(20)$ & $0.05(1)$ & 0.22 (4) & 15.8 (4) & 1.95 (9) & $0.13(9)$ & 99.8 & 0.256 \\
\hline \multirow[t]{5}{*}{ RP15 } & $\mathrm{gl}$ & 17 & $58.6(8)$ & $0.40(6)$ & $16.3(9)$ & $4.45(32)$ & $0.20(4)$ & $0.90(15)$ & $4.64(70)$ & $2.44(23)$ & $1.81(14)$ & 89.7 & 0.264 \\
\hline & amph & 7 & 41.9 (3) & $1.61(32)$ & 13.1 (3) & $17.5(11)$ & $0.41(5)$ & $9.9(5)$ & $10.3(4)$ & $1.86(4)$ & $0.65(3)$ & 97.1 & 0.502 \\
\hline & plg & 12 & $46.2(9)$ & 0.02 & $33.6(5)$ & $0.71(24)$ & $0.03(2)$ & 0.09 (7) & $16.9(5)$ & $1.61(30)$ & $0.10(5)$ & 99.2 & 0.189 \\
\hline & ilm & 5 & $0.22(15)$ & 50.9 (2) & 0.31 & 43.9 (5) & 1.03 (2) & 2.27 (4) & 0.08 (2) & 0.04 (3) & $<0.01$ & 98.7 & 0.084 \\
\hline & spl & 5 & $0.12(8)$ & $0.16(0)$ & $58.1(18)$ & $35.6(7)$ & 0.54 & $4.94(53)$ & $0.19(9)$ & $<0.01$ & $<0.01$ & 99.6 & 0.198 \\
\hline
\end{tabular}


Table 3 continued

\begin{tabular}{llllllllllllll}
\hline Run no. & Phase & $n$ & $\mathrm{SiO}_{2}$ & $\mathrm{TiO}_{2}$ & $\mathrm{Al}_{2} \mathrm{O} 3$ & $\mathrm{FeO}_{\text {tot }}$ & $\mathrm{MnO}$ & $\mathrm{MgO}$ & $\mathrm{CaO}$ & $\mathrm{Na}_{2} \mathrm{O}$ & $\mathrm{K}_{2} \mathrm{O}$ & $\mathrm{Total}$ & $\mathrm{Mg \#}$ \\
\hline $\mathrm{RP} 40$ & $\mathrm{gl}$ & 17 & $62.5(9)$ & $0.23(5)$ & $14.7(3)$ & $3.35(36)$ & $0.16(6)$ & $0.42(8)$ & $3.56(25)$ & $2.84(25)$ & $2.12(5)$ & 89.9 & 0.182 \\
& $\mathrm{amph}$ & 10 & $41.9(7)$ & $1.40(21)$ & $12.4(8)$ & $19.5(14)$ & $0.49(15)$ & $7.3(9)$ & $10.4(4)$ & $1.92(17)$ & $0.58(9)$ & 96.4 & 0.414 \\
& $\mathrm{plg}$ & 8 & $48.7(10)$ & $0.05(2)$ & $32.6(9)$ & $1.12(34)$ & $0.04(2)$ & $0.06(4)$ & $15.7(6)$ & $1.81(18)$ & $0.17(5)$ & 100.3 & 0.087 \\
& $\mathrm{ilm}$ & 8 & $0.11(9)$ & $47.8(11)$ & $0.33(4)$ & $49.3(8)$ & $0.98(8)$ & $1.24(2)$ & $0.10(2)$ & $<0.02$ & $<0.01$ & 99.8 & 0.043 \\
\hline
\end{tabular}

Units in parentheses indicate standard deviation from average analyses, in terms of significant digits and should be read as, e.g. $53.0 \pm 0.8 \mathrm{wt} \%$

Table 4 FT-IR results on starting materials synthesized at $1.6 \mathrm{GPa}, 1150^{\circ} \mathrm{C}$ for 30 minutes

\begin{tabular}{llllllllllllll}
\hline $\begin{array}{l}\text { Starting } \\
\text { material }\end{array}$ & $\begin{array}{l}\text { Run } \\
\text { No. }\end{array}$ & $\begin{array}{l}\mathrm{H}_{2} \mathrm{O} \\
\text { initial }\end{array}$ & $r(\mathrm{~g} / \mathrm{cm}-3)$ & $t \mathrm{H}_{2} \mathrm{O}^{\mathrm{a}}$ & Mol. abs. & $\mathrm{H}_{2} \mathrm{O}^{\mathrm{a}}$ & $t \mathrm{H}_{2} \mathrm{O}^{\mathrm{b}}$ & Mol. abs. & $\mathrm{H}_{2} \mathrm{O}^{\mathrm{b}}$ & $\mathrm{H}_{2} \mathrm{O}^{\mathrm{c}}$ & $t \mathrm{CO}_{2}$ & $\mathrm{Mol}^{\mathrm{abs}}{ }^{\mathrm{e}}$ & $\mathrm{CO}_{2}$ \\
\hline F8a-8c & PU 979 & 8.14 & 2.65 & 145 & 0.62 & $6.3(35)$ & 24 & 63 & $6.17(13)$ & $\sim 6.2$ & 24 & 305 & $0.89(2)$ \\
F8a-6c & PU 980 & 6.14 & 2.65 & 103 & 0.62 & $9.6(3)$ & 33 & 63 & $9.4(6)$ & 33 & 305 & $0.88(3)$ \\
F8a-4c & PU 981 & 4.14 & 2.65 & 46 & 0.62 & $7.7(3)$ & 28 & 63 & $7.3(3)$ & $\sim 6.8$ & 28 & 305 & $0.93(3)$ \\
\hline
\end{tabular}

All FT-IR results correspond to an average of four measurements at distinct locations

$t$ thickness of the glass wafer (in mm) used for $\mathrm{H}_{2} \mathrm{O}$ total, $\mathrm{H}_{2} \mathrm{O}$ molecular and $\mathrm{CO}_{2}$ measurements, respectively; $r$ calculated density of hydrous silicate glasses

${ }^{a} \mathrm{H}_{2} \mathrm{O}$ is calculated from the molecular $\mathrm{H}_{2} \mathrm{O}$ band at $5,200 \mathrm{~cm}^{-1}$

b $\mathrm{H}_{2} \mathrm{O}$ is calculated from the total $\mathrm{H}_{2} \mathrm{O}$ band at $3,530 \mathrm{~cm}^{-1}$

${ }^{c} \mathrm{H}_{2} \mathrm{O}$ determined by Raman spectroscopy

d The molar absorptivity of $\mathrm{H}_{2} \mathrm{O}$ molecular bands at $5,200 \mathrm{~cm}^{-1}$, and total $\mathrm{H}_{2} \mathrm{O}$ at $3,530 \mathrm{~cm}^{-1}$ was determinated by Dixon et al. (1995) and Newmann et al. (2000), respectively

e The molar absorptivity of the carbonate bands at $1,430 \mathrm{~cm}^{-1}(3051 / \mathrm{mol} \mathrm{cm})$ was calculated for an andesitic composition using the parameterization of Dixon and Pan (1995)

the peak heights for the $\mathrm{OH} / \mathrm{H}_{2} \mathrm{O}$ band intensity (around $3,500 \mathrm{~cm}^{-1}$ ) compared to the intensity ratio of the low frequency (LF, $400-600 \mathrm{~cm}^{-1}$ ) over the high frequency (HF, 800-1,200 $\mathrm{cm}^{-1}$ ) T-O bands corrected with an algorithm taking into account the composition (polymerization) dependence of the $\mathrm{LF} / \mathrm{HF}$ ratio as a function of $\mathrm{NBO} / \mathrm{T}$ (non-bridging oxygens per tetrahedrally coordinated cation, Mysen 1988). Estimated accuracy of the $\mathrm{H}_{2} \mathrm{O}$ determination using the described analytical procedure based on standard reproducibility and variability is in the order 0.5-1.0 wt $\% \mathrm{H}_{2} \mathrm{O}$. Results of quantitative $\mathrm{H}_{2} \mathrm{O}$ determinations of all experimental runs are listed in Table 2, and a comparison of $\mathrm{H}_{2} \mathrm{O}$ determinations by FTIR and Raman spectroscopy are shown in Table 4. The good agreement between FT-IR and Raman spectroscopic measurements suggests that the $\mathrm{H}_{2} \mathrm{O}$ contents of experimental runs as determined by Raman spectroscopy are probably accurate to $\pm 20 \%$.

\section{Experimental results}

$\mathrm{H}_{2} \mathrm{O}$-undersaturated experiments were conducted at 0.8 and $1.2 \mathrm{GPa}$ in a temperature range of $800-1,000^{\circ} \mathrm{C}$, and
$\mathrm{H}_{2} \mathrm{O}$ contents of 4,6 and 8 wt $\%$. For simplicity, we will refer to low-pressure experiments for those performed at $0.8 \mathrm{GPa}$, and to high-pressure experiments for those at 1.2 GPa. From the 25 equilibrium crystallization experiments reported in this study, nine have been performed with initial $\mathrm{H}_{2} \mathrm{O}$ contents of $8 \mathrm{wt} \%$, eight with $6 \mathrm{wt} \% \mathrm{H}_{2} \mathrm{O}$, and eight with $4 \mathrm{wt} \% \mathrm{H}_{2} \mathrm{O}$. Phases identified in the experiments include garnet (grt), clinopyroxene (cpx), orthopyroxene (opx), amphibole (amph), plagioclase (plg), ilmenite (ilm), spinel (spl) and liquid (liq). Generally, quench-free glasses (50-100 $\mu \mathrm{m}$ pools) were obtained for experiments at temperatures exceeding $900^{\circ} \mathrm{C}$, while at lower temperatures, the degree of crystallization increases and quench-free areas are usually smaller than $50 \mu \mathrm{m}$. Average liquid and solid phase major element compositions with their standard deviations are reported in Table 3. Quantitative measurements and BSE images reveal homogeneous glasses (Fig. 2). Inspection of BSE images showed that many of the experimental glasses contain relatively large and abundant vesicles of tens of micrometer in diameter, indicating that the majority of the experimental runs were vapor-saturated with a $\mathrm{CO}_{2}-\mathrm{H}_{2} \mathrm{O}$ fluid, consistent with the relatively high initial $\mathrm{CO}_{2}$ contents. These observations are summarized in Table 2 . 
Fig. 3 Isobaric T-X $\left(\mathrm{H}_{2} \mathrm{O}\right)$ phase diagrams for andesitic liquids a $0.8 \mathrm{GPa}$, and $\mathbf{b}$ $1.2 \mathrm{GPa}$. Dashed lines show the phase in/out boundaries derived from this study. Note that garnet-in at $0.8 \mathrm{GPa}$ is assumed. garnet $G r t$, clinopyroxene $C p x$, orthopyroxene $O p x$, amphibole Amph, plagioclase $\mathrm{Plg}$, ilmenite Ilm, spinel $\mathrm{Spl}$, liquid $\mathrm{Liq}$

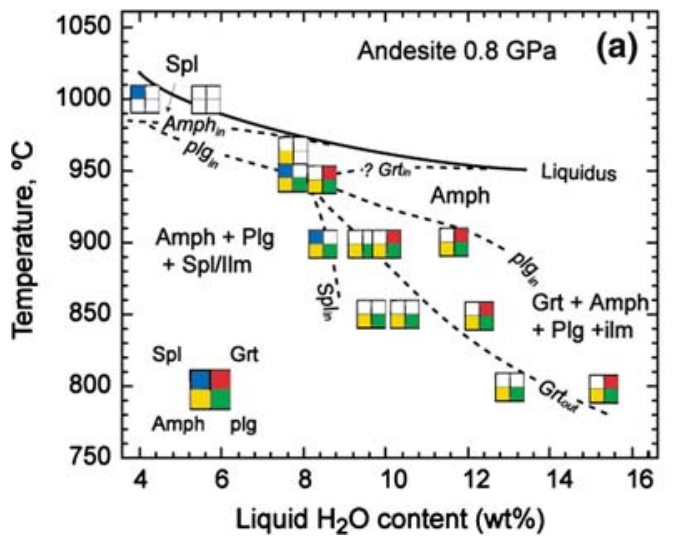

Homogeneous and euhedral to subhedral crystals exhibit no significant major element zoning as illustrated by the small relative errors on the major element composition (Table 3 ). The grain size of garnet can reach up to $100 \mu \mathrm{m}$ in diameter; amphibole and plagioclase are usually between 10 and $30 \mu \mathrm{m}$ in size, whereas pyroxenes and oxides tend to be smaller than $10 \mu \mathrm{m}$. Phase proportions are calculated by mass balance using respective bulk compositions (Table 1) and experimental phase compositions (Table 3) and a least squares regression routine implemented in the $\mathrm{EXCEL}^{\circledR}$ program. The principal phase relations at 0.8 and 1.2 $\mathrm{GPa}$ are illustrated in $\mathrm{T}-\mathrm{X}_{\mathrm{H}_{2} \mathrm{O}}$ diagrams in Fig. 3.

Phase relations at $0.8 \mathrm{GPa}$

At low-pressure (Fig. 3a), the liquidus temperature of the andesite is located below $1,000^{\circ} \mathrm{C}$ at $8 \mathrm{wt} \% \mathrm{H}_{2} \mathrm{O}$ and above $1,000^{\circ} \mathrm{C}$ at 6 and $4 \mathrm{wt} \% \cdot \mathrm{H}_{2} \mathrm{O}$. At $8 \mathrm{wt} \% \mathrm{H}_{2} \mathrm{O}$, the melt fraction $(f)$ decreases from 1 to 0.49 with decreasing temperature from 1,000 to $800^{\circ} \mathrm{C}$. Amphibole is the (first) liquidus phase and appears between $1,000^{\circ}$ and $950^{\circ} \mathrm{C}$ followed by garnet, plagioclase and ilmenite at $900^{\circ} \mathrm{C}$. The assemblage composed of garnet, amphibole, plagioclase and ilmenite remains stable down to temperatures of $800^{\circ} \mathrm{C}$ (Table 2 for details on phase proportions). With decreasing melt fraction, the amphibole/plagioclase ratio decreases slightly. This is observed in all series at $0.8 \mathrm{GPa}$. At $6 \mathrm{wt} \%$ $\mathrm{H}_{2} \mathrm{O}, f$ decreases from 0.8 to 0.45 with decreasing temperature $\left(950-800^{\circ} \mathrm{C}\right)$; garnet, amphibole, plagioclase and ilmenite constitute the liquidus phases at $950^{\circ} \mathrm{C}$ down to temperatures of $850^{\circ} \mathrm{C}$. At $800^{\circ} \mathrm{C}$, garnet disappeared, and amphibole, plagioclase and ilmenite form the phase assemblage. At $4 \mathrm{wt} \% \mathrm{H}_{2} \mathrm{O}, f$ decreases from 0.97 to 0.53 with decreasing temperature $\left(1,000-850^{\circ} \mathrm{C}\right)$. In this series, garnet is not present at any temperature examined. As noted by Helz (1982), the thermal stability of amphibole increases and the field of amphibole plus liquid expands (in basaltic systems) with increasing $\mathrm{H}_{2} \mathrm{O}$ contents, consistent with our results.
Phase relations at $1.2 \mathrm{GPa}$

At high-pressure (Fig 3b) and $8 \mathrm{wt} \% \mathrm{H}_{2} \mathrm{O}$, the melt fraction decreases systematically from 1 to 0.65 with decreasing temperature from 1,000 to $850^{\circ} \mathrm{C}$. The liquidus at these conditions is located around $1,000^{\circ} \mathrm{C},(f=1)$ with garnet and amphibole as the first liquidus phases observed at $950^{\circ} \mathrm{C}$, followed by plagioclase at $850^{\circ} \mathrm{C}$. Traces of ilmenite can be found $(<0.01 \mathrm{wt} \%)$. At $6 \mathrm{wt} \% \mathrm{H}_{2} \mathrm{O}, f$ decreases from 0.93 to 0.76 with decreasing temperature $\left(1,000-900^{\circ} \mathrm{C}\right)$; the liquidus temperature is above $1,000^{\circ} \mathrm{C}$. The first liquidus phase is garnet at $1,000^{\circ} \mathrm{C}$, followed by clinopyroxene and traces of amphibole at $950^{\circ} \mathrm{C}$. Amphibole is abundant at $900^{\circ} \mathrm{C}(14.9 \mathrm{wt} \%)$, suggesting that the cpx-amphibole reaction boundary is close to $950^{\circ} \mathrm{C}$, (Fig. 3b). The stability field of clinopyroxene at $6 \mathrm{wt} \%$ $\mathrm{H}_{2} \mathrm{O}$ is limited to the $900^{\circ} \mathrm{C}$ experiment. At high-pressure and $4 \mathrm{wt} \% \mathrm{H}_{2} \mathrm{O}, f$ decreases from 0.91 to 0.33 with decreasing temperature $\left(1,000-850^{\circ} \mathrm{C}\right)$; the liquidus temperature is above $1,000^{\circ} \mathrm{C}$. Garnet and cpx are the first liquidus phase at $1,000^{\circ} \mathrm{C}$ followed by cpx at $950^{\circ} \mathrm{C}$ and by plagioclase together with two pyroxenes and trace amounts of ilmenite $(0.8 \mathrm{wt} \%)$ at $900^{\circ} \mathrm{C}$. At $850^{\circ} \mathrm{C}$, garnet, amphibole and plagioclase are the crystallizing phases. We conclude that at high-pressure $(1.2 \mathrm{GPa})$ garnet is an important phase over the entire range of conditions (800$1,000^{\circ} \mathrm{C}, 4-8 \mathrm{wt} \% \mathrm{H}_{2} \mathrm{O}$ ). The stability field of amphibole is dependent on the $\mathrm{H}_{2} \mathrm{O}$ content in the melt: it is stable at high $\mathrm{H}_{2} \mathrm{O}$ contents to high temperatures and extends to low temperature-low $\mathrm{H}_{2} \mathrm{O}$ contents $\left(850^{\circ} \mathrm{C}, 4 \mathrm{wt} \% \mathrm{H}_{2} \mathrm{O}\right)$. On the contrary, increasing $\mathrm{H}_{2} \mathrm{O}$ contents shift plagioclase crystallization to lower temperatures, as observed in many other experimental studies (e.g. Sisson and Grove 1993a). The stability field of clinopyroxene is small compared to the other phases and is restricted to high temperatures exceeding $900^{\circ} \mathrm{C}$ and to medium to low $\mathrm{H}_{2} \mathrm{O}$-contents (4-6 $\mathrm{wt} \%$ ). Orthopyroxene appears only at $900^{\circ} \mathrm{C}$ and $4 \mathrm{wt} \%$ $\mathrm{H}_{2} \mathrm{O}$. Ilmenite and spinel are present all over the studied range as minor phases $(<0.4 \mathrm{wt} \%)$. For the following 
section on mineral compositions and related discussions, we have exclusively utilized those runs that suffered none or only minimal $\mathrm{H}_{2} \mathrm{O}$ loss (e.g. rp15 and rp13). Since the $\mathrm{H}_{2} \mathrm{O}$ content is one of the main variables that control the phase relations, we have reported experiments, which have suffered minor water loss, rp43 and rp88, with the perspective that an experiment with $8 \mathrm{wt} \%$ initial $\mathrm{H}_{2} \mathrm{O}$ that suffered minor $\mathrm{H}_{2} \mathrm{O}$-loss mimics an experiment with $\sim 5$ wt\% initial $\mathrm{H}_{2} \mathrm{O}$ (rp 43).

\section{Mineral compositions}

\section{Garnet}

Endmember compositions of the experimental garnets are illustrated in Fig. 4 and compared to data from basaltic systems (Alonso-Perez 2006; Müntener et al. 2001) and natural occurrences from island arcs. For a given pyrope content, low-pressure garnets are generally richer in almandine + spessartine and lower in grossular component than high-pressure ones. The effect of $\mathrm{H}_{2} \mathrm{O}$ on garnet compositions is not dramatic, but results in a tendency of increasing grossular content with increasing amounts of dissolved $\mathrm{H}_{2} \mathrm{O}$ in the liquid. Garnet compositions of our

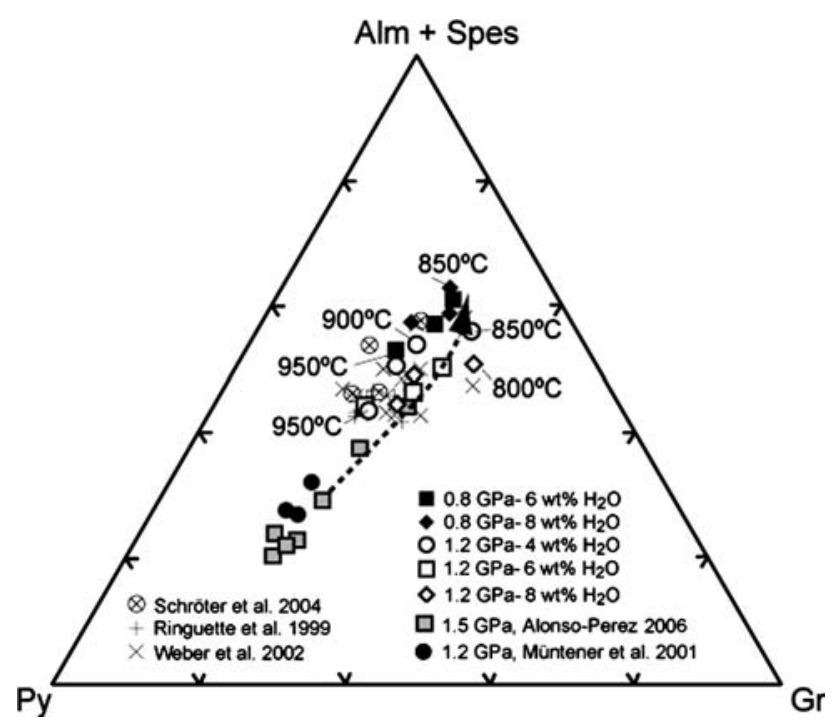

Fig. 4 Experimental garnet composition derived from this study ( 0.8 and 1.2 GPa) compared to basaltic systems (Alonso-Perez 2006; Müntener et al. 2001) and to natural occurrences from island arc systems (Schroter et al. 2004; Ringuette et al. 1999; Weber et al. 2002). Garnet end-member compositions are calculated based on $\mathrm{X}$-site occupancy $\left(\mathrm{Ca}, \mathrm{Fe}^{2+}, \mathrm{Mn}, \mathrm{Mg}\right)$. Note the shift towards the almandine-spessartine join for low-pressure $(0.8 \mathrm{GPa})$ garnets (full symbols) with respect to the high-pressure $(1.2 \mathrm{GPa})$ garnets (open symbols). Arrow indicates evolution of garnet compositions during isobaric cooling of the Jijal complex, Kohistan island arc (Müntener et al., unpublished data) study resemble those of "group C" (Coleman et al. 1965, garnets present in granulites) and are similar to granulite garnets commonly found, but not restricted to, subduction environments, such as those in Northern Fiordland, New Zealand (Schröter et al. 2004), deep-crustal xenoliths form the Northern Andes (Weber et al. 2002) or garnet cumulates from the Jijal complex, Kohistan arc (Ringuette et al. 1999, Müntener et al. unpublished data). Variations of garnet $\mathrm{Mg} \#$ expressed as the molar $\mathrm{Mg} /\left(\mathrm{Mg}+\mathrm{Fe}_{\text {tot }}\right)$ with decreasing temperature are positively correlated with decreasing $\mathrm{Mg} \#$ of the silicate liquid, and increasing degree of crystallization, independent of the initial $\mathrm{H}_{2} \mathrm{O}$ content.

\section{Amphibole}

Low-pressure amphiboles at $8 \mathrm{wt} \% \mathrm{H}_{2} \mathrm{O}$ vary from pargasite to Tschermakitic-hornblende (Leake 1978) with decreasing temperature from 950 to $800^{\circ} \mathrm{C}$. The $\mathrm{Mg} \#$ of amphibole decreases from 0.57 to 0.37 and shows a positive correlation with temperature for variable $\mathrm{H}_{2} \mathrm{O}$ contents (Fig. 5a). However, with the substantial increase of $\mathrm{Fe}-\mathrm{Ti}$ oxide crystallization in experiments at $800^{\circ} \mathrm{C}$ the Mg\# remains approximately constant. Thus, coexisting minerals have an important control on the $\mathrm{Mg} \#$ of amphibole. In low-pressure experiments with $4 \mathrm{wt} \%$ dissolved $\mathrm{H}_{2} \mathrm{O}$, garnet is absent and amphibole coexists with plagioclase and minor amounts of $\mathrm{Fe}$-Ti-oxides, while at higher $\mathrm{H}_{2} \mathrm{O}$ contents, garnet is the major coexisting $\mathrm{Fe}-$ $\mathrm{Mg}$ phase. This is reflected by the intermediate position of the $4 \mathrm{wt} \% \mathrm{H}_{2} \mathrm{O}$ line, between those of 8 and $6 \mathrm{wt} \% \mathrm{H}_{2} \mathrm{O}$, respectively (Fig. 5b). The much higher modal abundance of garnet at $8 \mathrm{wt} \% \mathrm{H}_{2} \mathrm{O}$ at $0.8 \mathrm{GPa}(3.8-10 \mathrm{wt} \%)$ as opposed to very small amounts $(0.1-1.3 \mathrm{wt} \%)$ at $6 \mathrm{wt} \%$, coupled with the low degree of crystallization shifts amphiboles from the $8 \mathrm{wt} \% \mathrm{H}_{2} \mathrm{O}$ experiments to much higher $\mathrm{Mg \#}$ because considerable amounts of $\mathrm{Fe}$ is accommodated in garnet that exhibits rather low $\mathrm{Mg} \#$ of 0.2-0.3 (Table 3).

High-pressure amphiboles are ferroan-pargasite at 8 and $6 \mathrm{wt} \% \mathrm{H}_{2} \mathrm{O}$ and Tschermakite at $4 \mathrm{wt} \% \mathrm{H}_{2} \mathrm{O}$ and $850^{\circ} \mathrm{C}$. The amphibole $\mathrm{Mg} \#$ decreases from 0.60 to 0.45 with decreasing temperature from 950 to $850^{\circ} \mathrm{C}$ and $\mathrm{H}_{2} \mathrm{O}$ contents from 8 to 4 wt\% (Fig. 5a). Thus, at low and highpressure conditions a systematic decrease of $\mathrm{Mg \#}$ with temperature is observed. This is illustrated in Fig. 5b, where amphibole and coexisting liquid $\mathrm{Mg \#}$ are plotted together with data from the literature. The $\mathrm{Fe}-\mathrm{Mg}$ exchange $\left(K_{\mathrm{d}}^{\sum \mathrm{Fe} / \mathrm{Mg}}\right)$ between amphibole and liquid derived from this study range between 0.29 and 0.47 and with an average value of $0.38 \pm 0.04$ Fig. $5 b$ ), in agreement with Sisson and Grove (1993a). Moreover, no 

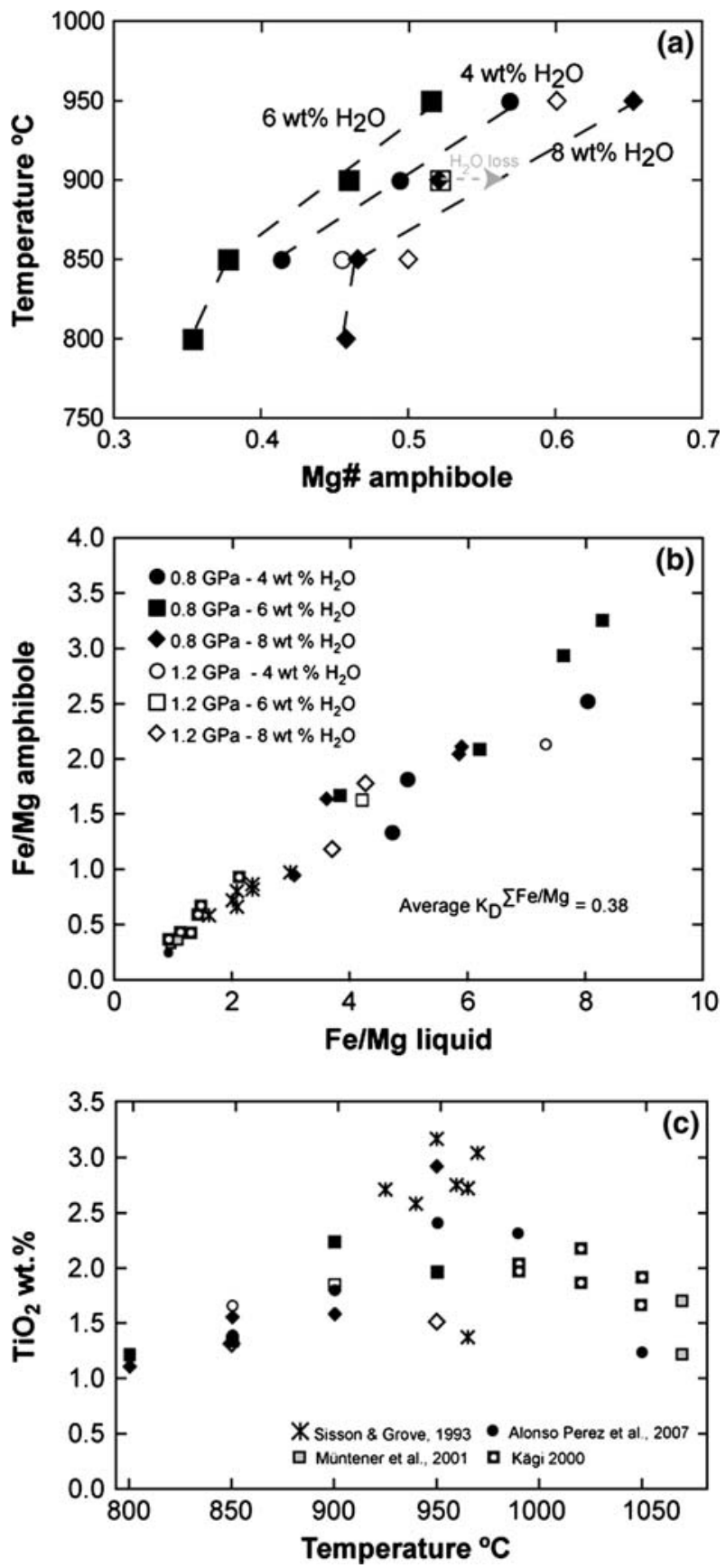

Fig. 5 Amphibole $\mathrm{Mg \#}$ variations as a function of temperature (a), $\mathrm{Fe}-\mathrm{Mg}$ exchange $\left(\mathrm{K}_{\mathrm{d}}^{\sum \mathrm{Fe} / \mathrm{Mg}}\right)$ between amphibole and liquid (b), and $\mathrm{TiO}_{2} \mathrm{wt} \%$ versus temperature for experimental amphiboles (c). $\left(\mathrm{K}_{\mathrm{d}}^{\sum \mathrm{Fe} / \mathrm{Mg}}\right)$ values range between 0.29 and 0.47 with an average value of $0.38 \pm 0.04$, in agreement with Sisson and Grove (1993a). Lines in a indicate smooth trends for all experiments except for those with substantial $\mathrm{Fe}-\mathrm{Ti}$ oxide crystallization at $800^{\circ} \mathrm{C}$. Note the shift of the $\mathrm{Mg} \#$ at $8 \mathrm{wt} \% \mathrm{H}_{2} \mathrm{O}$ and $900^{\circ} \mathrm{C}$ close to the $4 \mathrm{wt} \% \mathrm{H}_{2} \mathrm{O}$ line (rp43) consistent with some water loss

systematic correlation with pressure and a slight decrease in the $K_{\mathrm{d}}^{\sum \mathrm{Fe} / \mathrm{Mg}}$ with decreasing temperature can be observed. A positive correlation of increasing $\mathrm{TiO}_{2}$

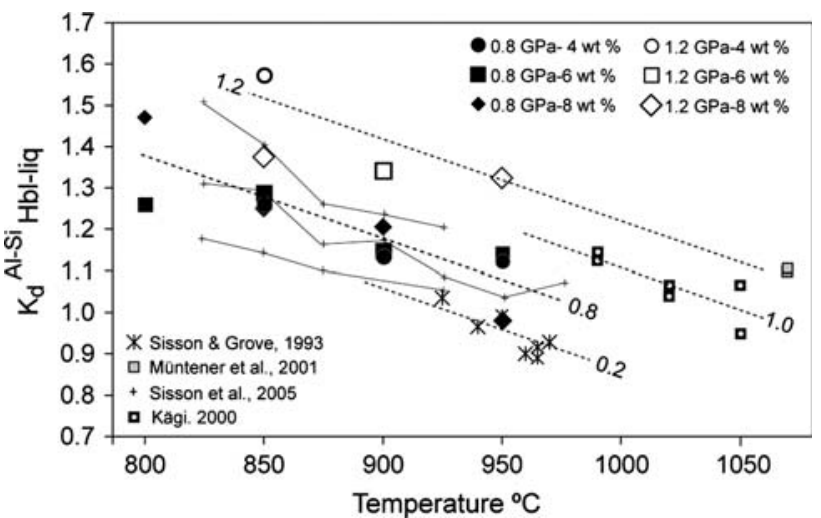

Fig. 6 Molar Al-Si exchange $\left(K_{\mathrm{d}}^{\mathrm{Al} / \mathrm{Si}}\right)$ between experimental amphiboles and liquids versus temperature. Dashed lines are eye-ball fits to different experimental series at different pressures. Solid lines connect experiments on three different compositions at $0.7 \mathrm{GPa}$ and $\mathrm{fO}_{2}$ similar to our study (Sisson et al. 2005). Positive pressure dependence for a given composition can be observed. High-pressure amphiboles of this study show a higher $K_{\mathrm{d}}^{\mathrm{Al}-\mathrm{Si}}$ ratio $\left(1.2 \mathrm{GPa}, K_{\mathrm{d}}=1.4\right)$ than the lower-pressure $\left(0.8 \mathrm{GPa}, K_{\mathrm{d}}=1.2\right)$ and the $0.2 \mathrm{GPa}$ hydrous experiments $\left(K_{\mathrm{d}}=0.94\right)$ of Sisson and Grove (1993a). However, experiments on 3 different compositions at a given pressure and $f \mathrm{O}_{2}$ similar to this study (QFM and BN experiments of Sisson et al. 2005) indicate that the $K_{\mathrm{d}}$ is also strongly composition dependent

contents with increasing temperature (Fig. 5c) can be monitored in andesitic bulk compositions up to $950^{\circ} \mathrm{C}$, similar to previous studies on $\mathrm{TiO}_{2}$ in amphibole (Otten 1984; Sisson and Grove 1993a). However, at higher temperatures in basaltic systems the $\mathrm{TiO}_{2}$ content slightly decreases with increasing temperature indicating incompatible behavior of $\mathrm{Ti}$ in the absence of coexisting $\mathrm{Fe}-\mathrm{Ti}$ oxides. The Al-Si exchange coefficient $\left(K_{\mathrm{d}}^{\mathrm{Al} / \mathrm{Si}}\right)$ between amphibole and liquids is plotted against temperature (Fig. 6) together with experimental amphibole/glass pairs from $\mathrm{H}_{2} \mathrm{O}$ saturated low-to intermediate pressure experiments (0.2 GPa) (Sisson and Grove 1993a; Sisson et al. 2005) and from $\mathrm{H}_{2} \mathrm{O}$ undersatured experiments on basaltic to andesitic systems Müntener et al. 2001; Kägi 2000). Although a temperature dependence of the $K_{\mathrm{d}}^{\mathrm{Al} / \mathrm{Si}}$ between amphibole and liquid is apparent, the data suggest that the $K_{\mathrm{d}}^{\mathrm{Al} / \mathrm{Si}}$ is also pressure and/or composition dependent. Contour lines for high pressure experiments of a given composition show generally higher $\left(K_{\mathrm{d}}^{\mathrm{Al} / \mathrm{Si}}\right)$ at a given temperature, than for low pressure experiments.

\section{Plagioclase}

The variation of the exchange $K_{\mathrm{d}}^{\mathrm{Ca}-\mathrm{Na}}$ between plagioclase and liquid as a function of liquid $\mathrm{H}_{2} \mathrm{O}$ content is illustrated in Fig. 7. The anorthite content of plagioclase is uniformly high for the low pressure experimental series $\left(X_{\mathrm{An}}\right.$ ranging from 0.8 to 0.88 , Table 3 ) and the $K_{\mathrm{d}}$ 's vary between 3.5 


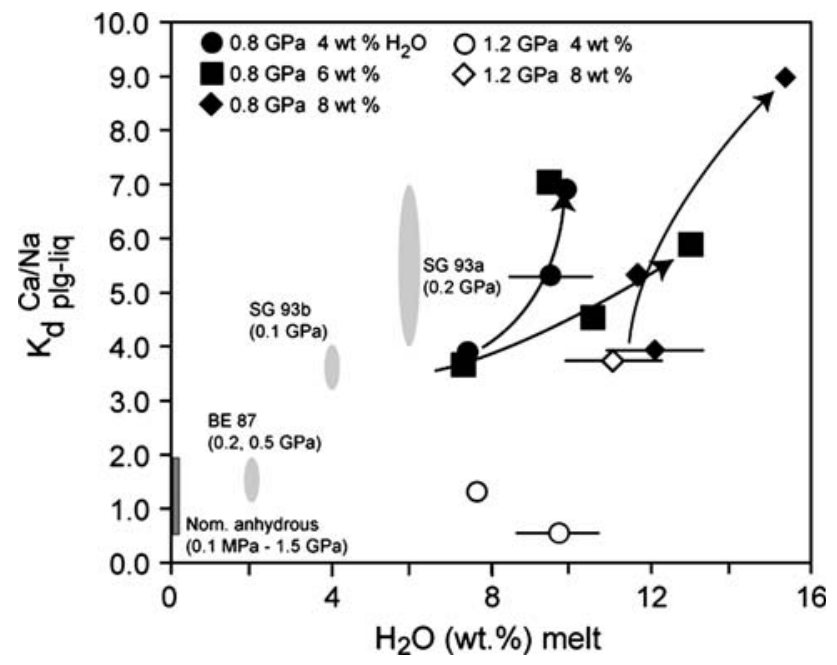

Fig. $7 \mathrm{H}_{2} \mathrm{O}$ contents of andesite liquids determined by Raman spectroscopy (Table 2) versus $\mathrm{Ca} / \mathrm{Na}$ partitioning between plagioclase

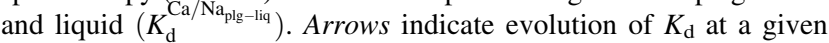
initial $\mathrm{H}_{2} \mathrm{O}$ content with decreasing temperature. Error bars indicate $\pm 1 \mathrm{wt} \%$ error on the absolute $\mathrm{H}_{2} \mathrm{O}$ content, as determined by Raman spectroscopy. Shaded fields indicate variations of $K_{\mathrm{d}}$ in $\mathrm{H}_{2} \mathrm{O}$ saturated and undersaturated experiments at low pressures: SG93a, $0.2 \mathrm{GPa}$ : Sisson and Grove (1993a), SG93b, 0.1 GPa: (Sisson and Grove 1993b) BE87, (Baker and Eggler 1987). $K_{\mathrm{d}}$ 's from nominally anhydrous experiments from $0.01 \mathrm{MPa}$ to $1.5 \mathrm{GPa}$ range from 0.5 to 2 (data from Baker and Eggler 1987; Bartels et al. 1991; Kinzler and Grove 1992; Draper and Johnston 1992; Villiger et al. 2004)

and 9. In a series of $0.2 \mathrm{GPa}_{2} \mathrm{O}$-saturated experiments, it was shown that the exchange $K_{\mathrm{d}}$ progressively increases with melt $\mathrm{H}_{2} \mathrm{O}$ content (Sisson and Grove 1993a). However, our results show that the $K_{\mathrm{d}}$ 's do not linearly increase at $\mathrm{H}_{2} \mathrm{O}$ undersaturated conditions. For each bulk $\mathrm{H}_{2} \mathrm{O}$ content, there is a positive correlation of the exchange $K_{\mathrm{d}}$ with liquid $\mathrm{H}_{2} \mathrm{O}$, much like the results from Yoder (1968). Hence, other parameters, such as partitioning of $\mathrm{Na}$ between plagioclase and coexisting amphibole $(\mathrm{Ca} /$ $\mathrm{Na}_{\text {amp }}<\mathrm{Ca} / \mathrm{Na}_{\text {plg }}$ ) are probably responsible for the further increase in $K_{\mathrm{d}}^{\mathrm{Ca}-\mathrm{Na}}$ with decreasing temperature and hence increasing $\mathrm{H}_{2} \mathrm{O}$ content in the melt. The exchange $K_{\mathrm{d}}$ of most of our experiments is substantially higher than in nominally anhydrous experiments between $0.1 \mathrm{MPa}$ and $1.5 \mathrm{GPa}$ (Fig. 7).

\section{Clinopyroxene}

Clinopyroxene was only observed in three experiments at $1.2 \mathrm{GPa}, 950$ and $900^{\circ} \mathrm{C}$, and at relatively low liquid $\mathrm{H}_{2} \mathrm{O}$ contents between 6 and $4 \mathrm{wt} \%$. The clinopyroxene is high $\mathrm{Al}_{2} \mathrm{O}_{3}$ diopside (7.6-9.2 wt\%). $\mathrm{Mg} \#$ and Ca-Tschermak's component range between 0.5 and 0.6 , and 0.08 and 0.14 , respectively.
Oxides

In all but three experiments containing $\mathrm{Fe}$-Ti-oxides, the oxide phase is ilmenite with an average composition of $41.0 \mathrm{wt} \% \mathrm{TiO}_{2}$, and $48.3 \mathrm{wt} \% \mathrm{FeO}$ (all $\mathrm{Fe}$ as $\mathrm{Fe}^{2+}$ ). The $\mathrm{MgO}$ content decreases from $3.3 \mathrm{wt} \%$ at $950^{\circ} \mathrm{C}$ to about 1 wt $\%$ at $800^{\circ} \mathrm{C}$ suggesting a temperature dependent incorporation of $\mathrm{Mg}$ into the ilmenite structure. Experiments rp67, rp14 and rp15 (0.8 GPa, 4wt $\mathrm{H}_{2} \mathrm{O}$, and 1,000 $900^{\circ} \mathrm{C}$, respectively) contain hercynitic spinel with $\mathrm{Mg \#}$ ranging from 0.41 to 0.2 .

\section{Melt compositions}

To evaluate the effects of different solid assemblages on the compositional evolution of experimental liquids during differentiation, we separately evaluate the low- and highpressure experimental series. Silica increases progressively with increasing differentiation (Fig. 8) from andesitic to dacitic and rhyolitic compositions (TAS diagram, Le Bas et al. 1986). $\mathrm{Mg}$-\# of derivative liquids systematically decreases from 0.41 to 0.17 with decreasing temperature from 1,000 to $800^{\circ} \mathrm{C}$ (Fig. 9). In general, high-pressure derivative liquids have higher $\mathrm{Mg \#}$ at a given temperature than low-pressure ones. The variability of four major elements (wt \%, on a volatile-free base) as a function of the Mg\# are illustrated in Fig. 9: $\mathrm{SiO}_{2}$ and $\mathrm{K}_{2} \mathrm{O}$ increase from 56.4 to $76.5 \mathrm{wt} \%$ and from 1.4 to $2.8 \mathrm{wt} \%$, respectively, whereas $\mathrm{CaO}$ and $\mathrm{TiO}_{2}$ decreases from 7.2 to $2.9 \mathrm{wt} \%$ and from 0.94 to $0.13 \mathrm{wt} \%$,

The quenched glass compositions at 0.8 and $1.2 \mathrm{GPa}$ are simplified by recasting liquid compositions into mineral

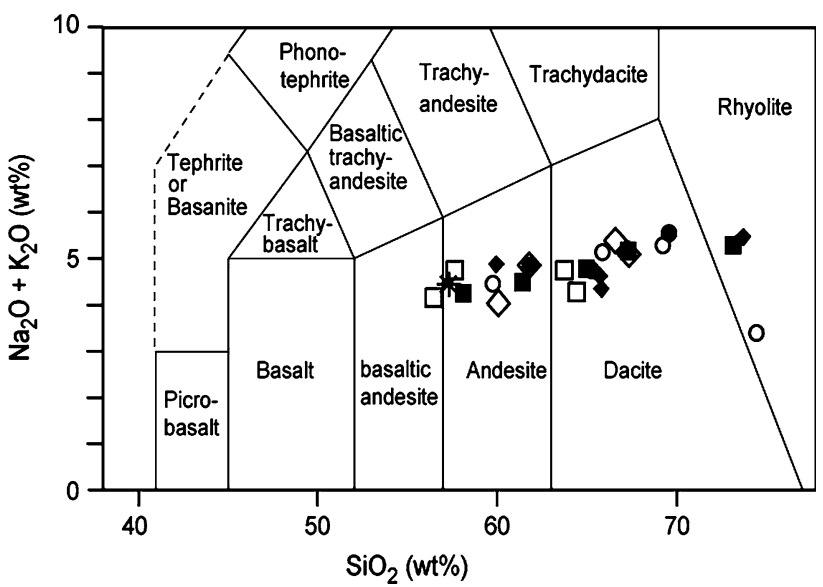

Fig. 8 A portion of the total alkali $\left(\mathrm{Na}_{2} \mathrm{O}+\mathrm{K}_{2} \mathrm{O}\right)$ vs silica $\left(\mathrm{SiO}_{2}\right)$ diagram (TAS, Le Bas et al. 1986), illustrating the compositional evolution of experimental liquids, recalculated on an anhydrous basis. Derivative liquids cover the range of basaltic andesite-andesitedacite-rhyolite 
Fig. 9 Derivative liquid major element compositions versus $\mathrm{Mg \# .} \mathrm{SiO}_{2}(\mathbf{a})$ and $\mathrm{K}_{2} \mathrm{O}(\mathbf{b})$ increase, whereas $\mathrm{CaO}(\mathbf{c})$ and $\mathrm{TiO}_{2}$ (d) decrease, with decreasing $\mathrm{Mg \#}$
Fig. 10 Liquid lines of descent at 0.8 and $1.2 \mathrm{GPa}$ projected into the Olivine-Cpx-Quartz pseudoternary system after Grove et al. (1992). The quenched glass compositions were recalculated into mineral end-member components and projected from spinel, plagioclase and $\mathrm{K}$-feldspar onto the Ol-Cpx-Qtz plane of the basalt tetrahedron. Note that most of the derivative compositions are peraluminous (corundum-normative), a feature that has often been cited as evidence for crustal melting (Conrad et al. 1988)
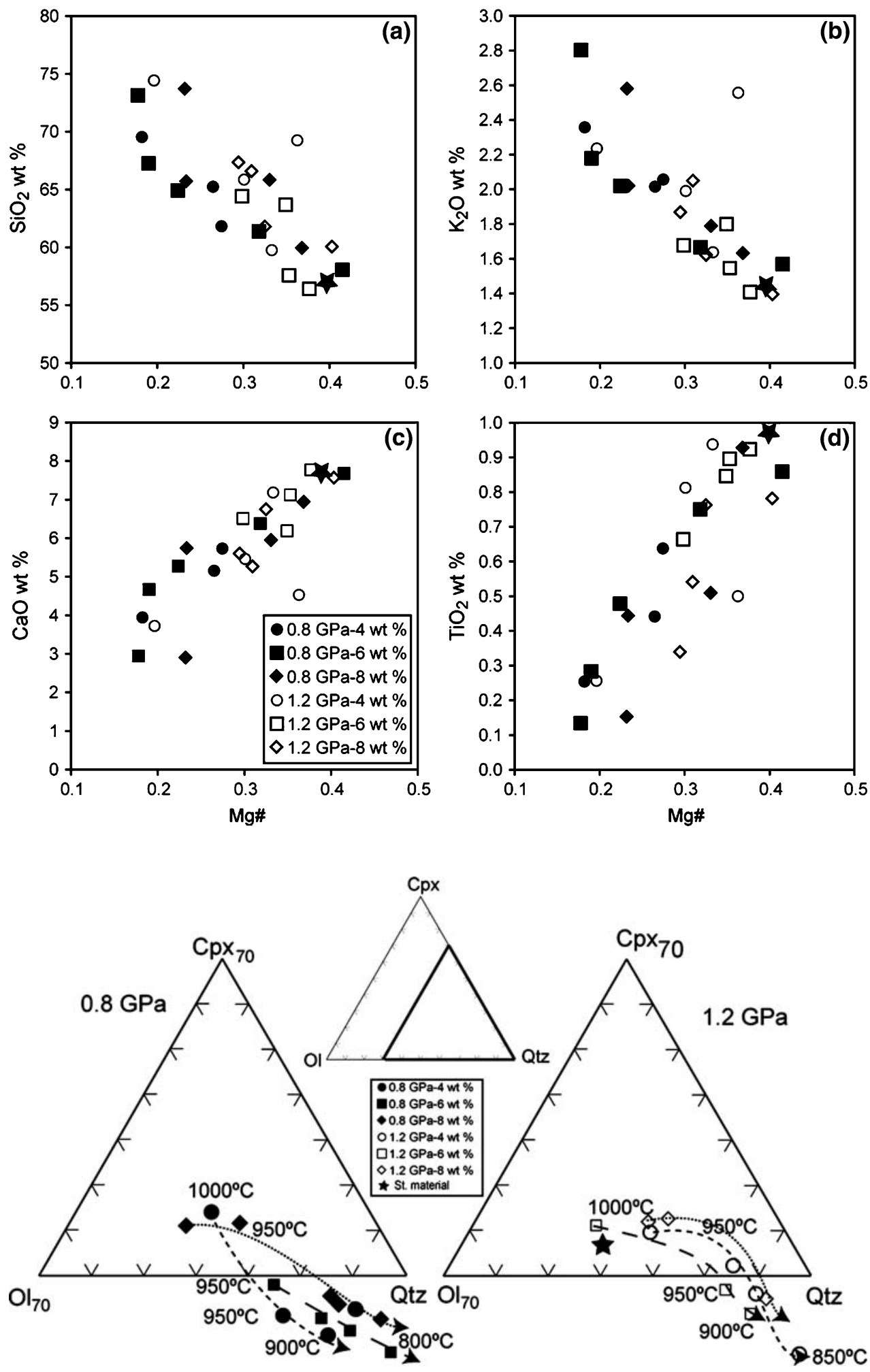

components and projecting them into the pseudo-ternary olivine-cpx-quartz system of the basalt tetrahedron (Fig. 10). The diagrams are projections through plagioclase and spinel (Grove et al. 1992). All derivative liquids produced in this study are qtz-normative. At $0.8 \mathrm{GPa}$
(Fig. 10a), liquids evolve to per-aluminous compositions mainly because of amphibole- dominated crystalline assemblages, except at the highest temperature $\left(\geq 950^{\circ} \mathrm{C}\right)$ close to the liquidus, and hence close to the original metaaluminous starting andesite composition. Liquid evolution 
is similar at $1.2 \mathrm{GPa}$ (Fig. 10b), with a change from metaaluminous to peraluminous compositions between 950 and $900^{\circ} \mathrm{C}$; higher modal proportions of garnet only slightly retards the evolution to peraluminous compositions. The overall liquid evolution is dominated by amphibole extraction that plots towards the left-side of the ol-cpx-qtz ternary, outside the triangle at positive olivine and cpx and negative Qtz coordinates (nepheline normative), driving residual liquids toward the lower-right corner into the corundum-normative field.

\section{Discussion}

Garnet and amphibole phase relations at pressures relevant for arc lower crust

In this study we have explored the roles of $\mathrm{H}_{2} \mathrm{O}$ and pressure at lower crustal conditions on the stability of the most important phases in a hydrous andesite liquid that was derived by fractional crystallization at $1.0 \mathrm{GPa}$ from a precursor picrobasaltic mantle-derived liquid. The principal phases we found are garnet, amphibole, clinopyroxene and plagioclase. We have identified rather larger stability fields of garnet at $1.2 \mathrm{GPa}$ and still had small amounts of almandine-rich garnet stable at $0.8 \mathrm{GPa}$. Garnet stability is clearly linked to the $\mathrm{H}_{2} \mathrm{O}$-content in the silicate liquid: At $0.8 \mathrm{GPa}$ the modal amounts of garnet increase with increasing $\mathrm{H}_{2} \mathrm{O}$ contents. The modal amount of garnet decreases with decreasing temperatures at the expense of increasing amphibole and plagioclase implying a reaction relation of the type garnet $+\operatorname{liquid}_{1}=\mathrm{amph}+\mathrm{plg}+$ liquid $_{2}$. At $1.2 \mathrm{GPa}$ the situation is somewhat different, as garnet reaches considerably larger maximal modal amounts, coexisting with cpx, at both 4 and $6 \mathrm{wt} \%$ initial $\mathrm{H}_{2} \mathrm{O}$ content, just at the onset of amphibole crystallization and rapidly decreases thereafter, indicating a peritectic relationship of the type: garnet $+\mathrm{cpx}+$ liquid $=$ amphibole. At $8 \mathrm{wt} \% \mathrm{H}_{2} \mathrm{O}$ the amount of amphibole at higher temperature is considerably larger and, as a consequence, the amount of garnet is reduced. A prime result of this study is that garnet is an igneous phase in typical hydrous, arc-tholeiitic to calc-alkaline derivative liquids at conditions corresponding to a magma reservoir located in the lower crust or at the crust-mantle boundary in convergentplate margin settings $(25-40 \mathrm{~km})$ and hence, its presence as a fractionating phase affecting both major and trace element contents of derivative liquids cannot a priori be attributed to partial melting of (solid) crustal source rocks and/or to melting of the subducting slab.

Estimated crustal thickness in active continental margin settings, range from approximately $70 \mathrm{~km}$ in the Andes, to $45 \mathrm{~km}$ in western North America, to 30-35 in Alaska (Gill
1981). The phase relationships established at 0.8 and $1.2 \mathrm{GPa}$, corresponding to a depth of approximately 25 $40 \mathrm{~km}$, are thus applicable to magmatic processes at conditions of a mature island arc setting or typical for active continental margin, and correspond to deep-crustal conditions or the crust-mantle boundary. The well-exposed crust-mantle section of the Jijal complex (Kohistan arc, Northern Pakistan) is composed of ultramafic olivine and cpx-rich cumulates (dunite, pyroxenite) at its base, overlain by amphibole and garnet-rich ultramafic rocks [garnethornblende pyroxenites, (cpx-) garnet-hornblendites and cpx- and amphibole-bearing garnetites] which in turn are overlain by garnet gabbros (Jan and Howie 1981; Ringuette et al. 1999). In the light of our new results the lithological succession of the upper part of the Jijal complex (starting with garnet and amphibole-rich ultramafic rocks) can be interpreted as fractionated products along a liquid line of decent of a hydrous basaltic andesite to andesite liquids at pressures between 0.8 and $1.2 \mathrm{GPa}$, temperatures in the range of $800-1,000^{\circ} \mathrm{C}, \mathrm{H}_{2} \mathrm{O}$ contents of $4-8 \mathrm{wt} \%$. Prograde dehydration melting is thus not required to explain arc root rock sequences (cf. Yoshino and Okudaira 2004; Garrido et al. 2006).

Phase control on meta-aluminous and peraluminous derivative liquids

Regarding the composition of our derivative liquids, there are two principal results: (1) the vast majority of our andesitic to rhyolitic liquids are peraluminous (corundumnormative, negative cpx-component). Peraluminous liquids containing garnet with less than 4 wt $\% \mathrm{CaO}$ have often been attributed to partial melting of sedimentary (pelitic) sources (Chappell et al. 1987; Conrad et al. 1988). However, such peraluminous granitoid rocks ranging from qtz-diorites to leucogranites are widespread in batholitic complexes (Ulmer et al. 1983; Blundy and Sparks 1992). Various experimental studies performed at our laboratory (Kägi 2000; Müntener and Ulmer 2006; Ulmer 2007 and unpublished data) in the range 0.5$1.5 \mathrm{GPa}$ on both equilibrium and fractional crystallization, and literature data at various pressures (Sisson and Grove 1993a; Müntener et al. 2001) indicate that peraluminous derivative liquids are mainly produced from meta-aluminous parental magmas at a restricted pressure range of about 0.7-1.2 GPa: At higher pressure (Alonso-Perez 2006) garnet precipitation inhibits the evolution towards peraluminous liquids, whereas at lower pressure dominance of An-rich plagioclase in hydrous systems keeps the liquids meta-aluminous. The 'critical' pressure range of about $0.7-1.2 \mathrm{GPa}$ is characterized by the dominance of amphibole in the fractionating assemblage that is only initially joined by cpx (at relatively low $\mathrm{H}_{2} \mathrm{O}$-contents) 
and the delay of plagioclase crystallization. This is in line with the original proposal by Cawthorn and Brown (1976) that identified amphibole as the dominant phase controlling the formation of corundum-normative liquids from meta-aluminous liquids. In summary, our phase equilibria clearly show that while garnet could crystallize from meta-aluminous andesite liquids under favorable conditions (high-pressure, high $\mathrm{H}_{2} \mathrm{O}$-contents) and will strongly control some trace elements in the derivative liquids (e.g. $\mathrm{Sr}, \mathrm{Y}, \mathrm{HREE}$ ), amphibole is the dominant phase controlling the major element characteristic of these magmas (high $\mathrm{SiO}_{2}$, peraluminous).

A second order effect related to amphibole fractionation is the observed $\mathrm{Al} / \mathrm{Si}$ partitioning behavior between amphibole and liquid (Fig. 6): With increasing pressure, the $K_{\mathrm{d}}^{\mathrm{Al} / \mathrm{Si}}\left((\mathrm{A} 1 / \mathrm{Si})_{\text {amph }} /(\mathrm{Al} / \mathrm{Si})_{\text {liq }}\right)$ increases and becomes larger than unity at $0.8 \mathrm{GPa}$. Therefore, it is probably not only garnet that drives derivative liquids at high pressure to meta-aluminous compositions (see above) but also amphibole becomes less efficient in crossing the peraluminous meta-aluminous compositional divide. However, given the large variability of $K_{\mathrm{d}}^{\mathrm{Al} / \mathrm{Si}}\left((\mathrm{A} 1 / \mathrm{Si})_{\mathrm{amph}} /\right.$ $\left.(\mathrm{Al} / \mathrm{Si})_{\text {liq }}\right)$ for different compositions at a given pressure (Fig. 6), these statements must remain qualitative at this point.

Calc-alkaline differentiation at high pressure?

The general evolution of derivative liquids in terms of tholeiitic versus calc-alkaline differentiation trends is

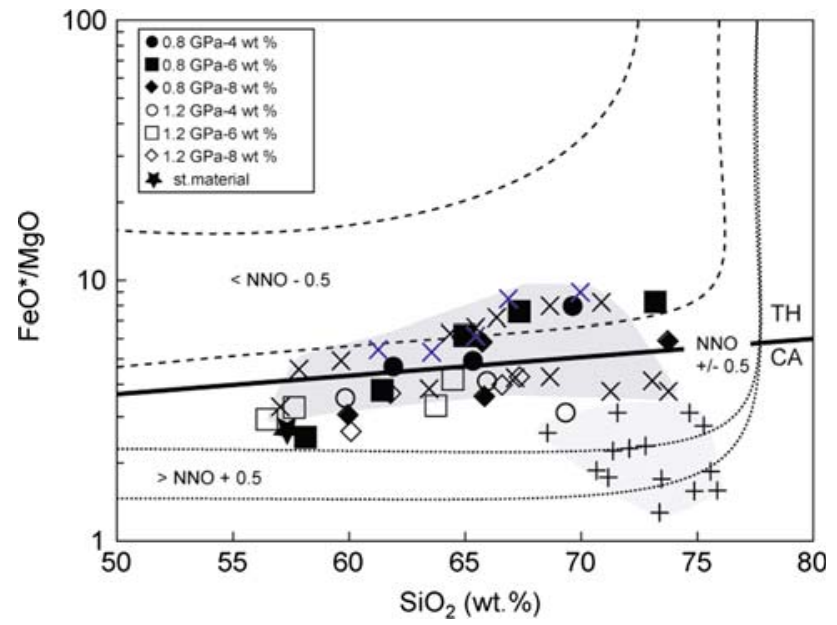

Fig. 11 Compositional characteristics of derivative liquids in an $\mathrm{FeO} * / \mathrm{MgO}$ versus $\mathrm{SiO}_{2}$ diagram depicting the calc-alkaline/tholeiitic dividing line from Miyashiro (1974) and $f \mathrm{O}_{2}$ fields from Sisson et al. (2005) for high-silica liquids. Crosses within light shaded field: highly oxidized experiments (buffered by $\mathrm{MnO}-\mathrm{Mn}_{3} \mathrm{O}_{4}$ ), diagonal crosses within darker shaded field: reduced experiments performed at QFM and BN from Sisson et al. (2005). Note that highly oxidized experiments tend to very low $\mathrm{FeO} * \mathrm{MgO}$ ratios illustrated in a binary $\mathrm{FeO} * / \mathrm{MgO}$ versus $\mathrm{SiO}_{2}$ wt\% diagram (Fig. 11), (Miyashiro 1974) together with the $\mathrm{fO}_{2}$ fields established by Sisson et al. (2005). High-pressure liquids remain in the calc-alkaline field, whereas lowpressure and high water content $\left(6-8\right.$ wt $\left.\% \mathrm{H}_{2} \mathrm{O}\right)$ liquids show a distinctly tholeiitic evolutionary trend. As shown before, the dominant plagioclase-amphibole crystallization at low-pressure, and high water contents $\left(6-8 \mathrm{wt} \% \mathrm{H}_{2} \mathrm{O}\right)$ drives derivative liquids to high $\mathrm{SiO}_{2}$ contents at moderately high $\mathrm{FeO}^{*} / \mathrm{MgO}$ values compared to the highpressure evolutionary trend, where garnet is an abundant phase that is not only silica-poor, but has a rather high $K_{\mathrm{d}}^{\mathrm{Fe}-\mathrm{Mg}_{\text {solid-liq }}}$ that produced high $\mathrm{SiO}_{2}$ and relatively low $\mathrm{FeO} * / \mathrm{MgO}$ characteristics of calc-alkaline products. The recent study of Sisson et al. (2005) concluded that in hydrous granite-rhyolite liquids at moderate pressure $(0.7 \mathrm{GPa})$ and an $\mathrm{fO}_{2}$ ranging from $\sim \mathrm{QFM}$ to $\mathrm{Ni}-\mathrm{NiO}$ +1.3 to +4 , the amount and composition of evolved liquids and coexisting mineral assemblages vary as functions of $\mathrm{fO}_{2}$ and temperature, with melt being more evolved at higher $\mathrm{fO}_{2}$. Furthermore, they argue that the best comparison of natural silicic magmas and experimentally produced liquids could be done in the discriminant diagram for tholeiitic versus calc-alkaline magmas (Miyashiro 1974), contoured for $\mathrm{fO}_{2}$. This is illustrated in Fig. 11, where we plotted some of the experimental data of Sisson et al. (2005), together with the experiments from this study. Their experiments with a similar setup to our experiments $\left(f \mathrm{O}_{2}\right.$ around $\mathrm{QFM}$ or $\left.\mathrm{BN}\right)$ closely overlap with our data, while their high $f_{2}$ experiments $\left(\mathrm{MnO}-\mathrm{Mn}_{3} \mathrm{O}_{4}\right.$ buffer: corresponding to QFM +4) plot on the high $\mathrm{SiO}_{2}-$ low $\mathrm{FeO} * / \mathrm{MgO}$ part of the diagram (Fig. 11). Data from this study are consistent with their conclusions; however, we point out that for a given $\mathrm{fO}_{2}$, pressure dependent phase equilibria are an important additional control on derivative granitic liquids.

An important effect of garnet, in addition to its influence on the trace element characteristics of derivative liquids, is its influence on the iron-magnesium ratio of derivative liquids. Garnet has a rather high $\mathrm{Fe} / \mathrm{Mg}$ solid/liquid partitioning coefficient $(0.78-0.92$ at $0.8-1.5 \mathrm{GPa})$ and hence tends to produce 'high-Mg' liquids (similar to magnetite) unlike amphibole that has a considerably lower $\mathrm{Fe} / \mathrm{Mg}$ solid/liquid $K_{\mathrm{d}}(0.32-0.45$ at $0.8-1.5 \mathrm{GPa})$, comparable to olivine and pyroxenes, and therefore, extracts $\mathrm{Mg}$ more efficiently, leading to lower $\mathrm{FeO}^{*} / \mathrm{MgO}$ liquids at a given $\mathrm{SiO}_{2}$-content (compare the high and low pressure series of Fig. 11). Bulk rock data from the plutonics of the Kohistan batholith (Jagoutz et al. submitted; Enggist personal communication 2008) indicates that high $\mathrm{Sr} / \mathrm{Y}$ granitoid rocks display lower $\mathrm{FeO} * \mathrm{MgO}$ at a given $\mathrm{SiO}_{2}$ than comparable rocks with low $\mathrm{Sr} / \mathrm{Y}$ ratios, supporting a potential role of garnet in producing "high-Mg\# granitoids". We tentatively 
propose that some leucogranites do not a priori represent 'crustal melts', but they might be formed directly by crystal fractionation at high pressures.

Absence of garnet leads to derivative liquids with rather high $\mathrm{FeO} * / \mathrm{MgO}$ contents at a given $\mathrm{SiO}_{2}$ content, whereas garnet suppresses this trend due to its high $\mathrm{Fe} / \mathrm{Mg}$ partition coefficient and keeps derivative liquids at rather low $\mathrm{FeO}^{*}$ / $\mathrm{MgO}$ ratios. At shallow pressure conditions (e.g. $<0.5 \mathrm{GPa}$ ), $f \mathrm{O}_{2}$ plays a dominant role by stabilizing $\mathrm{Fe}-\mathrm{Ti}-$ oxides (magnetite, hercynite, ulvospinel) to higher temperature and thus leading to higher modal amounts of these phases under oxidizing conditions (e.g. Sisson and Grove 1993a; Sisson et al. 2005). It should be mentioned that rather low amounts of $\mathrm{Fe}-\mathrm{Ti}$ oxide phases in high-pressure cumulates from island arc and active continental margins are a common feature: plagioclase-poor to plagioclase absent pyroxenites and hornblendites in the Jijal section of the Kohistan arc (Jan and Howie 1981; Ringuette et al. 1999; Müntener et al. unpublished data), and pyroxenitic to hornblenditic cumulates from the Adamello, Italy (Blundy and Sparks 1992; Ulmer et al. 1983); usually contain low modal amounts of Fe-Ti phases, unlike shallower gabbroic cumulates that contain abundant magnetite (Ulmer et al. 1983).

Acknowledgments We are grateful to $\mathrm{S}$. Pilet for measuring $\mathrm{CO}_{2}$ and $\mathrm{H}_{2} \mathrm{O}$ in the starting material by FT-IR at Caltech. We thank Bruno Scaillet and Alan Thompson for comments and M.J. Krawczynski who provided a helpful review that improved the paper. Insightful and constructive comments by T.W. Sisson forced us to think harder about $\mathrm{CO}_{2}$ and $\mathrm{fO}_{2}$ and substantially improved the overall content of the paper. This research was supported by the Swiss NSF (Grants nr. 2000-61894.00/1).

\section{References}

Allen JC, Boettcher AL (1978) Amphiboles in andesite and basalt: II. Stability as a function of P-T- $f \mathrm{H}_{2} \mathrm{O}-f \mathrm{O}_{2}$. Am Mineral 63:10741087

Allen JC, Boettcher AL (1983) The stability of amphibole in andesite and basalt at high pressures. Am Mineral 68:307-314

Allen JC, Boettcher AL, Marland G (1975) Amphiboles in andesite and basalt: I. Stability as a function of P-T- $f \mathrm{O}_{2}$. Am Min 60:1069-1085

Alonso-Perez R (2006) The role of garnet in the evolution of hydrous, calc-alkaline magmas: an experimental study at $0.8-1.5 \mathrm{GPa}$. $\mathrm{PhD}$ thesis, ETH Zurich, p 174

Atherton MP, Petford N (1993) Generation of sodium-rich magmas from newly underplated basaltic crust. Nature 362:144-146. doi: $10.1038 / 362144 \mathrm{a} 0$

Baker DR, Eggler DH (1987) Compositions of anhydrous and hydrous melts coexisting with plagioclase, augite, and olivine or low-Ca pyroxene from 1 atm to 8 kbar: application to the Aleutian volcanic center of Atka. Am Min 72:12-28

Bartels KS, Kinzler RJ, Grove TL (1991) High pressure phase relations of primitive high-alumina basalts from Medicine Lake volcano, northern California. Contrib Mineral Petrol 108:253270. doi:10.1007/BF00285935
Blundy JD, Sparks RSJ (1992) Petrogenesis of mafic inclusions in granitoid magmas of the Adamello massif, Italy. J Petrol 33:1039-1104

Blundy JD, Wood BJ (2003) Partitioning of trace elements between crystals and melts. Earth Planet Sci Lett 210:383-397

Bowen NL (1928) The evolution of igneous rocks. Princeton University Press, NJ

Burg JP, Bodinier JL, Chaudhry S, Hussain S, Dawood H (1998) Infra-arc mantle-crust transition and intra-arc mantle diapirs in the Kohistan complex (Pakistani Himalaya): petro-structural evidence. Terra Nova 10:74-80. doi:10.1046/j.1365-3121. 1998.00170.x

Cawthorn RG, Brown PA (1976) A model for the formation and crystallization of corundum-normative calcalkaline magmas through amphibole fractionation. J Geol 84:467-476

Cawthorn RG, O'Hara MJ (1976) Amphibole fractionation in calcalkaline magma genesis. Am J Sci 276:309-329

Chappell BW, White AJR, Wyborn D (1987) The importance of residual source material (restite) in granite petrogenesis. J Petrol 28(6):1111-1138

Coleman RG, Lee DE, Beatty LB, Brannock WW (1965) Eclogites and eclogites-their differences and similarities. Geol Soc Am Bull 76:483-508. doi:10.1130/0016-7606(1965)76[483:EAETDA] 2.0.CO;2

Conrad WK, Nicholls IA, Wall VJ (1988) Water-saturated and -undersaturated melting of metaluminous and peraluminous crustal compositions at $10 \mathrm{~kb}$ : evidence for the origin of silicic magmas in the Taupo Volcanic Zone, New Zealand, and other occurrences. J Petrol 29:765-803

Davidson J, Turner S, Handley H, Macpherson C, Dosseto A (2007) Amphibole 'sponge' in the arc crust. Geology 35:787-790. doi: 10.1130/G23637A.1

Day RA, Green TH, Smith IEM (1992) The origin and significance of garnet phenocrysts and garnet-bearing xenoliths in miocene calcalkaline volcanics from Northland, New Zealand. J Petrol 33:125-161

DeBari SM, Coleman RG (1989) Examination of the deep levels of an island arc: Evidence from the Tonsina ultramafic-mafic assemblage, Tonsina, Alaska. J Geophys Res 94:4373-4391. doi: 10.1029/JB094iB04p04373

Defant MJ, Drummond MS (1990) Derivation of some modern arc magmas by melting of young subducted lithosphere. Nature 347:662-665. doi:10.1038/347662a0

Di Muro A, Villemant B, Montagnac G, Scaillet B, Reynard B (2006) Quantification of water content and speciation in natural silicic glasses (phonolite, dacite, rhyolite) by confocal micro-Raman spectrometry. Geochim Cosmochim Acta 70:2868-2884. doi: 10.1016/j.gca.2006.02.016

Dixon JE, Pan V (1995) Determination of the molar absorptivity of dissolved carbonate in basanitic glass. Am Min 80:1339-1342

Dixon JE, Stolper EM, Holloway JR (1995) An experimental study of water and carbon dioxide solubilities in mid-ocean ridge basaltic liquids 1. Calibration and solubility models. J Petrol 36:16071631

Draper DS, Johnston AD (1992) Anhydrous PT phase relations of an aleutian high- $\mathrm{MgO}$ basalt: an investigation of the role of olivineliquid reaction in the generation of high-alumina basalts. Contrib Mineral Petrol 112:501-519. doi:10.1007/BF00310781

Ducea MN, Saleeby JB (1996) Buoyancy sources for a large, unrooted mountain range, the Sierra Nevada, California: evidence form xenolith thermobarometry. J Geophys Res 101(B4):8229-8244. doi:10.1029/95JB03452

Ducea MN, Saleeby JB (1998) The age and origin of a thick maficultramafic keel from beneath the Sierra Nevada batholith. Contrib Mineral Petrol 133:169-185. doi:10.1007/ s004100050445 
Evans BW, Vance JA (1987) Epidote phenocrysts in dacitic dikes, Boulder county, Colorado. Contrib Mineral Petrol 96:178-185. doi:10.1007/BF00375231

Fitton JG (1972) The genetic significance of almandine-pyrope phenocrysts in the calc-alkaline Borrowdale volcanic group, Northern England. Contrib Mineral Petrol 36:231-248. doi: 10.1007/BF00371434

Gaetani GA, Grove TL (1998) The influence of water on melting of mantle peridotite. Contrib Mineral Petrol 131:323-346. doi: 10.1007/s004100050396

Garrido CJ, Bodinier J-L, Burg J-P, Zeilinger G, Hussain SS, Dawood H, Chaudry N, Gervilla F (2006) Petrogenesis of mafic garnet granulite in the lower crust of the Kohistan Paleo-arc complex (Northern Pakistan): Implications for intra-crustal differentiation of island arcs and generation of continental crust. J Petrol 47:1872-1914. doi:10.1093/petrology/eg1030

Gill JB (1981) Orogenic Andesites and plate tectonics. Springer, Berlin, p 390

Green TH (1972) Crystallization of calc-alkaline andesite under controlled high pressure hydrous conditions. Contrib Mineral Petrol 34:150-166. doi:10.1007/BF00373770

Green TH (1992) Experimental phase equilibrium studies of garnetbearing I-type volcanics and high-level intrusives from Northland, New Zealand. Trans R Soc Edinb Earth Sci 83:429-438

Green TH, Ringwood AE (1968a) Genesis of the calc-alkaline igneous rock suite. Contrib Mineral Petrol 18:105-162. doi: 10.1007/BF00371806

Green TH, Ringwood AE (1968b) Origin of garnet phenocrysts in calc-alkaline rocks. Contrib Mineral Petrol 18:163-174. doi: 10.1007/BF00371807

Greene AR, DeBari SM, Kelemen PB, Blusztain J, Clift PD (2006) A detailed geochemical study of island arc crust: the Talkeetna arc section, south-central Alaska. J Petrol 47:1051-1093. doi: 10.1093/petrology/eg1002

Grove TL, Kinzler RJ, Bryan WB (1992) Fractionation of mid-ocean ridge basalt (MORB). In: Phipps Morgan J, Blackman DK, Sinton JM (eds) Mantle flow and melt generation at mid-ocean ridges, vol 71. American Geophysical Union, Washington, DC, pp 281-310

Hall LJ, Brodie J, Wood BJ, Carroll MR (2004) Iron and water losses from hydrous basalts contained in $\mathrm{Au}_{80} \mathrm{Pd}_{20}$ capsules at high pressure and temperature. Min Mag (Lond) 68:75-81. doi: 10.1180/0026461046810172

Harangi SZ, Downes H, Kosa L, Szabo C, Thirlwall MF, Mason PRD, Mattey DP (2001) Almandine Garnet in calc-alkaline volcanic rocks of the Northern Pannonian basin (Eastern Central Europe): Geochemistry, petrogenesis and geodynamic implications. J Petrol 42:1813-1843. doi:10.1093/petrology/42.10.1813

Helz RT (1982) Phase relations and compositions of amphiboles produced in studies of the melting behavior of rocks. In: Ribbe $\mathrm{P}$ (ed) Amphiboles; petrology and experimental phase relations. Mineralogical Society of America, Washington, DC, pp 279-353

Huang W-L, Wyllie PJ (1986) Phase relationships of gabbro-tonalitegranite-water at $15 \mathrm{kbar}$ with applications to differentiation and anatexis. Am Min 71:301-316

Jan MQ, Howie RA (1981) The mineralogy and geochemistry of the metamorphosed basic and ultrabasic rocks of the Jijal complex, Kohistan, NW Pakistan. J Petrol 22:85-126

Kägi R (2000) The liquid line of descent of hydrous, primary calcalkaline magmas under elevated pressure. An experimental approach. PhD thesis, ETH Zürich, p 100

Kägi R, Müntener O, Ulmer P, Ottolini L (2005) Piston cylinder experiments on $\mathrm{H}_{2} \mathrm{O}$ undersaturated Fe-bearing systems: an experimental setup approaching $f \mathrm{O}_{2}$ conditions of natural calcalkaline magmas. Am Min 90:708-717. doi:10.2138/am.2005. 1663
Kay S, Mahlburg KS, Kay RW (1985) Aleutian tholeiitic and calcalkaline magma series I: the mafic phenocrysts. Contrib Mineral Petrol 90:276-290. doi:10.1007/BF00378268

Kelemen PB, Hanghøj K, Greene AR (2003) One view of the geochemistry of subduction-related magmatic arcs, with an emphasis on primitive andesite and lower crust. In: Rudnick RL (ed) The crust. Elsevier, New York, pp 593-659

Kinzler RJ, Grove TL (1992) Primary magmas of mid-ocean ridge basalts 1. Experiments and methods. J Geophys Res 97:68856906. doi:10.1029/91JB02840

Kress VC, Carmichael ISE (1991) The compressibility of silicate liquids containing $\mathrm{Fe} 2 \mathrm{O} 3$ and the effect of composition, temperature, oxygen fugacity and pressure on their redox states. Contrib Mineral Petrol 108:82-92. doi:10.1007/BF00307328

Kushiro I (1987) A petrological model of the mantle wedge and lower crust in the Japanese island arcs. In: Mysen B (ed): Magmatic processes, physicochemical principles. Geochem Soc Spec Publ $1: 165-181$

Le Bas MJ, Lemaitre RW, Streckeisen A, Zanettin B (1986) A chemical classification diagram of volcanic rocks based on the total alkali silica diagram. J Petrol 27(3):745-750

Leake BE (1978) Nomenclature of amphiboles. Am Min 63:10231052

Mercier M, Di Muro A, Giordano D, Métrich N, Lesne P, Pichavant M, Scaillet B, Clocchiatti R, Montagnac G (2008) Influence of glass polymerization and oxidation on Micro-Raman water analysis in alumino-silicate glasses. Geochim Cosmochim Acta (in press)

Miyashiro A (1974) Volcanic rock series in island arcs and active continental margins. Am J Sci 274:321-355

Müntener O, Ulmer P (2006) Experimentally derived high-pressure cumulates from hydrous arc magmas and consequences for the seismic velocity structure of island arc crust. Geophys Res Lett 31:L21308. doi:10.1029/2006GL027629

Müntener O, Kelemen PB, Grove TL (2001) The role of $\mathrm{H}_{2} \mathrm{O}$ during crystallization of primitive arc magmas under uppermost mantle conditions and genesis of igneous pyroxenites: an experimental study. Contrib Mineral Petrol 141:643-658

Mysen BO (1988) Structure and properties of silicate melts. Elsevier, Amsterdam

Osborn EF (1959) Role of oxygen pressure in the crystallization and differentiation of basaltic magma. Am J Sci 257:609-647

Newman S, Stolper E, Stern R (2000) $\mathrm{H}_{2} \mathrm{O}$ and $\mathrm{CO}_{2}$ in magmas from the Mariana arc and back arc systems. Geochem Geophys Geosystems 1. doi:10.1029/1999GC000027

Otten MT (1984) The origin of brown hornblende in the Artfjället gabbro and dolerites. Contrib Mineral Petrol 86(2):189-199. doi: 10.1007/BF00381846

Pilet S, Baker M, Stolper EM (2008) Metasomatized lithosphere and the origin of alkaline lavas. Science 320:916-919. doi:10.1126/ science. 1156563

Ratajeski K, Sisson TW (1999) Loss of iron to gold capsules in rockmelting experiments. Am Min 84:1521-1527

Ringuette L, Martignole J, Windley BF (1999) Magmatic crystallization, isobaric cooling, and decompression of the garnetbearing assemblages of the Jijal sequence (Kohistan terrane, western Himalayas). Geology 27:139-142. doi:10.1130/00917613(1999)027<0139:MCICAD > 2.3.CO;2

Rudnick RL (1995) Making continental crust. Nature 378:571-577. doi: $10.1038 / 378571 \mathrm{a} 0$

Schroter FC, Stevenson JA, Daczko NR, Clarke GL, Pearson NJ, Klepeis KA (2004) Trace element partitioning during high-P partial melting and melt-rock interaction; an example from northern Fiordland, New Zealand. J Metamorph Geol 22(5):443457. doi:10.1111/j.1525-1314.2004.00525.x

Sisson TW, Grove TL (1993a) Experimental investigations of the role of $\mathrm{H}_{2} \mathrm{O}$ in calc-alkaline differentiation and subduction zone 
magmatism. Contrib Mineral Petrol 113:143-166. doi: 10.1007/BF00283225

Sisson TW, Grove TL (1993b) Temperatures and $\mathrm{H}_{2} \mathrm{O}$ contents of low $\mathrm{MgO}$ high-alumina basalts. Contrib Mineral Petrol 113:167-184. doi:10.1007/BF00283226

Sisson TW, Ratajeski K, Hankins WB, Glazner AF (2005) Voluminous granitic magmas from common basaltic sources. Contrib Mineral Petrol 148:635-661. doi:10.1007/s00410-004-0632-9

Stern CR, Huang W-L, Wyllie PJ (1975) Basalt-andesite-rhyolite$\mathrm{H}_{2} \mathrm{O}$ crystallization intervals with excess $\mathrm{H}_{2} \mathrm{O}$ and $\mathrm{H}_{2} \mathrm{O}$-undersaturated liquidus surfaces to 35 kilobars, with implications for magma genesis. Earth Planet Sci Lett 28:189-196. doi:10.1016/ 0012-821X(75)90226-5

Taylor SR (1967) The origin and growth of continents. Tectonophysics 4:17-34. doi:10.1016/0040-1951(67)90056-X

Tiepolo M, Vannucci R, Oberti R, Foley S, Bottazzi P, Zanetti A (2000) $\mathrm{Nb}$ and $\mathrm{Ta}$ incorporation and fractionation in titanian pargasite and kaersutite; crystal-chemical constraints and implications for natural systems. Earth Planet Sci Lett 176:185-201. doi:10.1016/S0012-821X(00)00004-2

Ulmer P (1986) Basische und ultrabasische Gesteine des Adamello (Provinzen Brescia und Trento, Norditalien). PhD thesis Nr. 8105. ETH Zürich

Ulmer P (1989a) High pressure phase equilibria of a calc-alkaline picro-basalt: Implications for the genesis of calc-alkaline magmas. Carnegie Inst Wash $\mathrm{Yb}$ 88:28-35

Ulmer P (1989b) Partitioning of High Field Strength Elements among olivine, pyroxenes, garnet and calc-alkaline picrobasalts: experimental results and an application. Carnegie Inst Wash $\mathrm{Yb}$ $88: 42-47$

Ulmer P (2007) Differentiation of mantle-derived calc-alkaline magmas at mid to lower crustal levels: experimental and petrologic constraints. Minerva 76:309-325
Ulmer P, Callegari E, Sonderegger UC (1983) Genesis of the mafic and ultramafic rocks and their genetical relations to the tonalitictrondhjemitic granitoids of the southern part of the Ademello Batholith, (Northern Italy). Mem Soc Geol Ital 26:171-222

Villiger S, Ulmer P, Müntener O, Thompson AB (2004) The liquid line of descent of anhydrous, mantle-derived, tholeiitic liquids by fractional and equilibrium crystallization-an experimental study at 1.0 GPa. J Petrol 45:2369-2388. doi:10.1093/petrology/ egh042

Villiger S, Ulmer P, Müntener O (2007) Equilibrium and fractional crystallization experiments at $0.7 \mathrm{GPa}$ : the effect of pressure on phase relations and liquid compositions of tholeiitic magmas. J Petrol 48:159-184. doi:10.1093/petrology/eg1058

Weber MBI, Tarney J, Kempton PD, Kent RW (2002) Crustal makeup of the Northern Andes; evidence based on deep crustal xenolith suites, Mercaderes, SW Colombia. In: Wörner G, Jaillard E (eds) Andean geodynamics. Elsevier, Amsterdam

Wilke M, Behrens H (1999) The dependence of the partitioning of iron and europium between plagioclase and hydrous tonalitic melt on oxygen fugacity. Contrib Mineral Petrol 137:102-114. doi: $10.1007 / \mathrm{s} 004100050585$

Yoder HS (1968) Albite-anorthite-quartz-water at 5 kbar. Carnegie Inst Wash Yb 66:477-478

Yoshino T, Okudaira T (2004) Crustal growth by magmatic accretion constrained by metamorphic p-T paths and thermal models of the Kohistan arc, NW Himalayas. J Petrol 45:2287-2302. doi: 10.1093/petrology/egh056

Zajacz Z, Halter W, Malfait W, Bachmann O, Bodnar RJ, Hirschmann MM, Mandeville CW, Morizet Y, Müntener O, Ulmer P, Webster J (2005) A composition-independent quantitative determination of the water content in silicate glasses and silicate melt inclusions by confocal Raman spectroscopy. Contrib Mineral Petrol 150:631-642. doi:10.1007/s00410-005-0040-9 